\title{
Synthesis of light-inducible and light-independent anthocyanins regulated by specific genes in grape 'Marselan' (V. vinifera L.)
}

\author{
Zong-Huan Ma ${ }^{1}$ ， Wen-Fang Li ${ }^{1}$ ， Juan Mao ${ }^{1}$ ， Wei Li ${ }^{1}$ ， Cun-Wu Zuo ${ }^{1}$, Xin Zhao ${ }^{1}$, Mohammed Mujitaba \\ Dawuda $^{1}$, Xing-Yun Shi ${ }^{2}$, Bai-Hong Chen ${ }^{\text {Corresp. } 1}$ \\ ${ }^{1}$ College of Horticulture, Gansu Agricultural University, Lanzhou, China \\ 2 Wuwei Academy of Forestry Science, Wuwei, China \\ Corresponding Author: Bai-Hong Chen \\ Email address: bhch@gsau.edu.cn
}

Anthocyanin is an important parameter for evaluating the quality of wine grapes. However, the effects of different light intensities on anthocyanin synthesis in grape berry skin and its regulation mechanisms are still unclear. In this experiment, clusters of wine grape cv. 'Marselan' were bagged using fruit bags with different light transmittance of $50 \%, 15 \%$, $5 \%$, and 0 , designated as treatment $A, B, C$ and $D$, respectively. Fruits that were not bagged were used as the control, designated as CK. The anthocyanin composition and concentration, as well as gene expression profiles in the berry skin were determined. The results showed that the degree of coloration of the berry skin reduced with the decrease of the light transmittance, and the veraison was postponed for 10 days in $D$ when compared with the CK. Total anthocyanin concentration in the berry skin treated with $D$ decreased by $51.50 \%$ compared with CK at the harvest stage. A total of 24 and 21 anthocyanins were detected in CK and D, respectively. Among them, Malvidin-3-O-coumaroylglucoside (trans) which showed a significant positive correlation with the total concentration of anthocyanins at the harvest stage $(r=0.775)$ and was not detected in $\mathrm{D}$, was presumed to be light-induced anthocyanin. Other anthocyanins which were both synthesized in CK and $D$ were considered to be light-independent anthocyanins. Among them, Malvidin-3-Ocoumaroylglucoside (cis) and Malvidin-3-O-acetylglucoside were typical representatives. Remarkably, the synthesis of light-inducible anthocyanins and light-independent anthocyanins were regulated by different candidate structural genes involved in flavonoid biosynthesis pathway and members of MYB and bHLH transcription factors. 
1 Synthesis of light-inducible and light-independent

2 anthocyanins regulated by specific genes in grape

3 'Marselan' ( $V$. vinifera L.)

4 Zong-Huan $\mathrm{Ma}^{1}$, Wen-Fang $\mathrm{Li}^{1}$, Juan $\mathrm{Mao}^{1}$, Wei $\mathrm{Li}^{1}$, Cun-Wu Zuo ${ }^{1}$, Xin Zhao ${ }^{1}$,

5 Mohammed Mujitaba Dawuda ${ }^{1}$, Xing-Yun Shi ${ }^{2}$ and Bai-Hong Chen ${ }^{1}$

6

$7{ }^{1}$ College of Horticulture, Gansu Agricultural University, Lanzhou, Gansu, China

$8 \quad{ }^{2}$ Wuwei Academy of Forestry Science, Wuwei, Gansu, China

9

10 Corresponding author

11

12 Bai-Hong Chen

13 Email address: bhch@gsau.edu.cn

14 College of Horticulture, Gansu Agricultural University, Lanzhou, Gansu, 730070, China

\section{Abstract}

17 Anthocyanin is an important parameter for evaluating the quality of wine grapes. However, the effects of different light intensities on anthocyanin synthesis in grape berry skin and its regulation mechanisms are still unclear. In this experiment, clusters of wine grape cv.

'Marselan' were bagged using fruit bags with different light transmittance of 50\%, 15\%, 5\%, and 0 , designated as treatment $\mathrm{A}, \mathrm{B}, \mathrm{C}$ and $\mathrm{D}$, respectively. Fruits that were not bagged were used as the control, designated as CK. The anthocyanin composition and concentration, as well as gene expression profiles in the berry skin were determined. The results showed that the degree of colorationof the berry skin reduced with the decrease of the light transmittance, and the veraison was postponed for 10 days in $\mathrm{D}$ when compared with the CK. Total anthocyanin 
26 concentration in the berry skin treated with D decreased by $51.50 \%$ compared with CK at the

27 harvest stage. A total of 24 and 21 anthocyanins were detected in CK and D, respectively.

28 Among them, Malvidin-3-O-coumaroylglucoside (trans) which showed a significant positive

29 correlation with the total concentration of anthocyanins at the harvest stage $(r=0.775)$ and

30 was not detected in D, was presumed to be light-induced anthocyanin. Other anthocyanins

31 which were both synthesized in CK and D were considered to be light-independent

32 anthocyanins. Among them, Malvidin-3-O-coumaroylglucoside (cis) and

33 Malvidin-3-O-acetylglucoside were typical representatives. Remarkably, the synthesis of

34 light-inducible anthocyanins and light-independent anthocyanins were regulated by different

35 candidate structural genes involved in flavonoid biosynthesis pathway and members of MYB 36 and bHLH transcription factors.

\section{Introduction}

Phenolic compounds play an important role in the sensory properties of wine (Quijada-Morín et al., 2012), and can be divided into two categories, flavonoids and nonflavonoids (Figueiredo-González et al., 2012; Pérez-Gregorio et al., 2014; Perez-Gregorio \& Simal-Gandara, 2017a, b). Anthocyanin is one of the important flavonoids and plays an important role in the formation of fruit color and quality (Bellincontro et al., 2010; Figueiredo-González et al., 2012a, b, c; Ilieva et al., 2016; Moro et al., 2017). The concentration and composition of anthocyanins during fruit ripening are related to the climate, soil, place of growing and cultivation (Ortega-Regules et al., 2006; Carbone et al., 2009; Figueiredo-González et al., 2013; Liang et al., 2014; Raventós \& Izquierdo-Pulido, 2014; Zhang et al., 2018;). Light is an important environmental factor affecting the synthesis of flavonoids in most plants (Guan et al., 2014; He et al., 2016). Therefore, it is important to study the effects of light and other environmental factors on anthocyanin synthesis and reveal 51 the regulation mechanism for improving fruit quality. 
52 Studies have shown that higher light intensity can promote the accumulation of anthocyanins in most plants (Maier et al., 2015). In 'Hakuho' peach (Prunus persica Batsch),

54 the area and intensity of the skin's red color were increased with increasing bag exposure to sunlight (Jia et al., 2015). In addition to light intensity, light quality can also affect the synthesis of anthocyanins. The previous studies showed that the anthocyanin concentrations under red and blue light treatment were higher than that in the dark conditions (Xu et al., 2014; Liu et al., 2015). UV-light alters concentrations of individual anthocyanins in grape berry skin (Crupi et al., 2013; Zoratti et al., 2014). In red pear cv. 'Red Zaosu', continuous illumination with blue light and red light increased the concentration of anthocyanins in the pericarp, and the blue light promoted the accumulation of anthocyanins more than the red light (Tao et al., 2018). The concentration and proportion of anthocyanin in strawberries (Fragaria $\times$ ananassa) treated with colored light-quality selective plastic films were significantly different from those of the control (Miao et al., 2016). Among these, the red and yellow films promoted anthocyanin accumulation, while the green and blue films decreased anthocyanin concentration.

The anthocyanin composition in the grape berry skin is also affected by cultivar, temperature and light conditions by altering the expression of genes involved in the flavonoid biosynthetic pathway (Azuma et al., 2012). Concentrations of total anthocyanins, pelargonidin-3-glucoside and pelargonidin-3-malonylglucoside in strawberries were significantly increased after blue and red light treatment (Zhang et al., 2018). In the veraison stage of grapes, the formation of color is mainly due to the synthesis of malvidin-based, and the anthocyanin concentration in grape berries at mature stage was significantly higher than that of the control after blue and red light irradiation (Kondo et al., 2014). Some studies suggested that the expression of flavanone $3^{\prime}-$ hydroxylase $\left(F 3^{\prime} H\right)$, flavonoid $3^{\prime} 5^{\prime}$ hydroxylase $\left(F 3^{\prime} 5^{\prime} H\right)$ and $O$-methyltransferases $(O M T)$ strongly affected the variation in anthocyanin composition 


\section{Manuscript to be reviewed}

77 (Castellarin \& Gaspero, 2007; Azuma et al., 2012). In the berry skin of grape cv. 'Guipu No.

78

6', the proportion of cyanidin-type (3'-substituted) anthocyanins was higher than that in

'Cabernet Sauvignon', which could be related to higher expression of F3'H (Cheng et al., 2017). F3'5'H is the key enzyme in delphinidin biosynthesis (Fukui et al., 2003), whereas $F 3^{\prime} H$ catalyzes the metabolism of naringenin to eriodictyol (Doostdar et al., 1995; Zhou et al., 2016). Light exclusion increased the ratio of dihydroxylated to trihydroxylated anthocyanins in grape, which in parallel with $F 3^{\prime} H$ and $F 3^{\prime} 5^{\prime} H$ transcript amounts (Guan et al., 2016).

Transcription factors encoding MYB-like and bHLH proteins appeared to modulate the expression of the structural genes involved in anthocyanin biosynthesis pathway (Lepiniec et al., 2006; Azuma et al., 2012). The apple MdMYB1/MdMYBA transcription factor has been shown in earlier studies to be a positive regulator of light-controlled anthocyanin biosynthesis (Takos et al., 2006; Ban et al., 2007; Lin-Wang et al., 2010). A new study found that MdMYB1 accumulates in light, but is degraded via a ubiquitindependent pathway in the dark (Li et al., 2012). Cominelli et al. (2008) reported that $b H L H$ genes analysed showed light induction, and their expression preceded that of the late structural genes, suggesting their possible role in light regulation of these structural genes (Cominelli et al., 2008).

Bagged apple fruits cannot be colored, and the removal of the fruit bags resulted in a rapid increase in anthocyanin concentration in the skin, which became red in a relatively short period of time (Wang et al., 2015). The leaf color of red lettuce (Lactuca sativa L.) developed poorly coloring grown at low light intensity (Zhang et al., 2018). However, several studies suggested that anthocyanin biosynthesis was not readily affected by sunlight in grape (Price et al., 1995; Downey et al., 2014). In addition, the difference of anthocyanin components in grape under different light intensity is still not clear. This study is based on the hypothesis that light-independent anthocyanins are dominant in grapes, and their synthesis is regulated by different genes. In this experiment, we analyzed the composition of anthocyanin in the berry

Peerj reviewing PDF | (2018:11:32873:1:1:NEW 23 Jan 2019) 
102 skin and the key genes in its metabolic pathway under fruit bags with different light

103 transmission properties to clarify the role of light in the regulation of anthocyanin synthesis in

104 wine grape cv. 'Marselan'.

105

\section{Materials \& Methods}

107 Plant materials and experimental design

108 Nine years old wine grape cv. 'Marselan' ochard belonging to the Wuwei Academy of Forestry

109 Science $\left(102^{\circ} 42^{\prime} \mathrm{E}, 38^{\circ} 02^{\prime} \mathrm{N}\right.$; altitude $1,632 \mathrm{~m}$; Gansu, China) was used for the present study.

110 The climate in this area corresponds to the warm temperate zone, with an average annual

111 temperature of $6.9^{\circ} \mathrm{C}$, an average annual rainfall of $191 \mathrm{~mm}$, an annual evaporation of 2,130

$112 \mathrm{~mm}$ and an sunshine duration of $2,720 \mathrm{~h}$. The frost-free period is more than 160 days, with long

113 duration of sunshine and large variations in temperature during production. The grape plants

114 were self-rooted and planted at $3 \times 0.5 \mathrm{~m}$ spacing. Seven adjacent vine rows, each containing

115 approximately 120 vines, were used for the experiment. Five were manipulated and measured,

116 and two were used as border rows, positioned between of experimental rows. Each plant was

117 pruned to allow only 5 branches and we also prune to allow 2 clusters per branch. Drip

118 irrigation with the volume of $4,800 \mathrm{~m}^{3} \cdot \mathrm{ha}^{-1}$ was applied. In addition, $\mathrm{N}, \mathrm{P}_{2} \mathrm{O}_{5}$ and $\mathrm{K}_{2} \mathrm{O}$ was

119 applied to $160 \mathrm{~kg} \cdot \mathrm{ha}^{-1}, 120 \mathrm{~kg} \cdot \mathrm{ha}^{-1}$ and $240 \mathrm{~kg} \cdot \mathrm{ha}^{-1}$, respectively. Clusters were bagged at 45

120 days after flowering and the bags were maintained until harvest. Four different bags (wood

121 pulp, Zhaofeng, Yantai, China), including one-layered white bags, stripe (yellow and brown)

122 bags, brown bags and two-layered bags (the inner layer was completely red and the outer bag

123 had black inner lining whiles the outside of the outer bag was brown), designated as A, B, C

124 and D, with light transmittance of 50\%, 15\%, 5\% and 0, respectively. The control (CK)

125 treatment consisted of fruits that were not bagged. Each of the 5 treatments had 90 clusters

126 from 30 grapevines, and each treatment was replicated three times. Berries were harvested at

PeerJ reviewing PDF | (2018:11:32873:1:1:NEW 23 Jan 2019) 
12790 (S1), 100 (S2) and 125 (S3) days after flowering, respectively. Each repetition was

128 harvested from three clusters, of which 10 berries were randomly selected from the base,

129 middle and top of the cluster, respectively. A total of 30 berries were randomly selected from

130 each replicate. Each treatment was replicated three times. The berry skin was quickly peeled

131 off, immediately frozen in liquid nitrogen and stored at $-80{ }^{\circ} \mathrm{C}$ for further analysis of

132 anthocyanin contents and composition. In addition, samples from S2 (CK1, A1, B1, C1 and D1)

133 and S3 (CK2, A2, B2, C2 and D2) were used for RNA-seq analysis.

135 Ultra - high performance liquid chromatography-tandem mass spectrometry

\section{6 (UHPLC-MS/MS) analysis of anthocyanins}

137 Total anthocyanin concentration was analyzed from each treatment according to the technique

138 described (Rabino \& Mancinelli, 1986) with some modifications. In brief, lyophilized berry

139 skin samples were finely ground with liquid nitrogen, and $1.0 \mathrm{~g}$ of powdered samples were 140 transferred to the extract solution $(20 \mathrm{~mL})$ containing methanol / hydrochloric acid mixture

141 (99:1 v/v) to extract anthocyanins at $4{ }^{\circ} \mathrm{C}$ for 24 hours. The mixture was incubated and then

142 centrifuged at $9,168 \mathrm{~g}$ at $4{ }^{\circ} \mathrm{C}$ for $20 \mathrm{~min}$. The supernatant was transferred into the cuvette, and

143 it concentrations at $530 \mathrm{~nm}$ and $657 \mathrm{~nm}$ were determined by spectrophotometer (Shimadzu,

144 Kyoto, Japan). A = OD $530-0.25 \mathrm{OD}_{657}$ was used to calculate the concentration of anthocyanins.

145 The extraction of total anthocyanin component was done as described by (He et al.,2016)

146 with some modifications. The berry skin is placed in mortar and ground with liquid nitrogen.

147 About $2.0 \mathrm{~g}$ of the powdered samples were added in $10 \mathrm{~mL}$ centrifuge tube with $8 \mathrm{~mL} 2 \%$

148 formic acid-methanol solution. After $10 \mathrm{~min}$ of ultrasonic oscillation, the extract was placed in

149 the dark at $25^{\circ} \mathrm{C}$ on $4 \mathrm{~g}$ for $30 \mathrm{~min}$, and centrifuged at $4{ }^{\circ} \mathrm{C}$ on $13,201 \mathrm{~g}$ for $10 \mathrm{~min}$. The

150 supernatant fraction was changed into $50 \mathrm{~mL}$ centrifuge tube and the residue was re-extracted

151 three times. The vacuum rotary evaporator (BUCH, USA) was used to vaporize the organic 
152 fraction at $40{ }^{\circ} \mathrm{C}$. Pour the residual parts into the active solid phase extraction cartridge

153 (Macclesfiled, UK). The residue was flushed twice with $5 \mathrm{~mL}$ water. After removing the

154 leachate, the solid phase extraction cartridge was eluted twice with $10 \mathrm{~mL}$ methanol. The

155 filtrate was collected and evaporated. Malvidin-3-O-glucoside as external standard quantitative,

156 mass concentration gradient was $0-50 \mathrm{mg} \cdot \mathrm{L}^{-1}$, other anthocyanins were calculated to be

157 equivalent to the concentration of Malvidin-3- $O$-glucoside.

158 UHPLC analysis was performed on an Agilent 1290 UHPLC system coupled with an

159 Agilent 6460 triple quadrupole mass spectrometer in positive ion mode $\left([\mathrm{M}+\mathrm{H}]^{+}\right)$. The

160 chromatographic column was $120 \mathrm{EC}-\mathrm{C} 18$ column $\left(150 \times 2.1 \mathrm{~mm}, 2.7 \mu \mathrm{m}, 40^{\circ} \mathrm{C}\right.$. $)$. The

161 mobile phases were filtered with $0.45 \mu \mathrm{m}$ degassed membrane filter in vacuum. Phase A

162 (formic acid : water was 0.5: $100 \mathrm{v} / \mathrm{v}$ ) and B (formic acid : methanol: acetonitrile was $0.5: 50$ :

$16350, \mathrm{v} / \mathrm{v})$. The gradient elution: $0 \mathrm{~min}, 90 \%$ of $\mathrm{A}, 10 \%$ of B; 0-28 min, $54 \%$ of $\mathrm{A}, 46 \%$ of B;

$16428-29 \min , 90 \%$ of $\mathrm{A}, 10 \%$ of $\mathrm{B} ; 29-34 \mathrm{~min}, 90 \%$ of $\mathrm{A}, 10 \%$ of $\mathrm{B}$. The sample volume was 3

$165 \mu \mathrm{L}$, the flow rate was $0.4 \mathrm{~mL} /$ minute, and a column temperature was $30^{\circ} \mathrm{C}$. Mass spectrometry

166 results were analyzed using Mass Lynx V4.1 software, and the identification of the material

167 structure was referenced to the standard substance as described by (Lopes-da-Silva et al.,

168 2002).

169

170 RNA isolation, cDNA library construction and transcriptome sequencing

171 The total RNA was extracted with RNA plant reagent (Real-Times Biotechnology, Beijing,

172 China) and evaluated with a $1 \%$ agarose gel stained with GoldView. The RNA quality and

173 quantity were assessed by using a Nanodrop 2000 Spectrophotometer and an Agilent 2100

174 Bioanalyzer (Agilent Technologies, Sangta Clara, CA, USA). For each berry skin treatment,

175 the RNA specimen of three randomly sampled individuals were aggregated as mixed samples.

176 These mixed samples were used for cDNA construction and RNA sequencing, which were 
177 completed by Guangzhou Sagene Bioinformation Technology Co. Ltd. The cDNA library was

178 constructed with $3 \mu \mathrm{g}$ RNA of each sample. To select DNA fragments of preferentially

179 150-200 bp in length, library fragments were purified with AMPure XP system (Beckman

180 Coulter, Beverly, MA, USA). Other details for the process of library construction was

181 described in Mao et al. (2018), and the 10 libraries were sequenced on an Illumina HiSeq 2000

182 platform.

183

184 RNA-seq data analysis

185 Raw reads were cleaned by removing the adapter sequences and the clean reads were aligned to

186 the grape reference genome (http://plants.ensembl.org/Vitis_vinifera/Info/Index) using the

187 program Tophat v2.0.943. During the detection process of differentially expressed genes

188 (DEGs), the absolute value of the $\log 2$ (fold change) with Fragments Per Kilobase Million

$189(\mathrm{FPKM}) \geq 1$ were used as the threshold to determine the significantly DEGs in this research.

190 The DEGs were analyzed by GOseq R software packages (Mao et al., 2018) and had a

191 significant enrichment effect on the modified Gene Ontology (GO) terms with corrected $P$

192 value $<0.05$. In order to get the detailed function of classification in different treatments,

193 software KOBAS was used to conduct a full enrichment test of different genes expressed in the

194 KEGG pathway. The pathways with Q value $\leq 0.01$ were considered significantly enriched

195 (Kanehisa et al., 2004; Xie et al., 2011).

196

197 Quantitative real-time PCR validation of RNA-seq data

198 To quantitatively determine the reliability of our transcriptional data, we monitored the

199 expression of 4 candidate DEGs using qRT-PCR. Specific primer pairs were designed as

200 shown in Table S1. The qRT-PCR was performed using the Roche Light Cycler96 Real-Time

201 Detection System (Roche) with SYBR Green PCR Master Mix (Takara). The thermal profile 
202 for SYBR Green I RT-PCR was $95{ }^{\circ} \mathrm{C}$ for $15 \mathrm{~min}$, followed by 40 cycles of $95^{\circ} \mathrm{C}$ for 10 s and

$20360{ }^{\circ} \mathrm{C}$ for 30 s and $72{ }^{\circ} \mathrm{C}$ for 30s. The reference gene $U B I\left(\mathrm{XM} \_002266714\right)$ was used as

204 internal reference. The comparative $2^{-\Delta \Delta \mathrm{CT}}$ method was used to analyze the expression levels of 205 the different genes (Livak \& Schmittgen, 2001). All of the samples were tested in triplicate, and 206 the experiments were performed on three biological replicates.

208 Statistical analysis

209 Statistical analyses were performed using analysis of variance (ANOVA) followed by

210 Duncan's new multiple range tests with SPSS version 17.0 (SPSS, Chicago, IL, USA). A

211 significance level of $p<0.05$ was applied. Correlation tests were performed using Pearson

212 product-moment correlation coeffcient (Pearson's $r$ ) with a two-tailed test.

214 Results

215 Effects of light intensity on veraison and total anthocyanin concentration in grape berry 216 skin

217 Results showed that different bagging treatments affected duration of color change in grape

218 berries (Fig. 1a). The veraison of A and CK occurred almost simultaneously, but the coloring 219 of B was 3 days later compared with CK. while that of C and D was delayed for10 days. The 220 color conversion rates of $\mathrm{CK}, \mathrm{A}$ and $\mathrm{B}$ were higher than $50 \%$ at $\mathrm{S} 1$, but there was no coloring in 221 the clusters of C and D. In addition, the clusters of CK and A were completely coloured at S2, 222 while C and D were just beginning to be coloured. However, berries from all treatments were 223 completely coloured and the appearance was not different at S3. The total anthocyanin concentration decreased with decreases in light transmittance of fruit bags from the three development stages, of which A, B, C and D were decreased by $10.10 \%, 19.23 \%, 37.07 \%$ 
226 and $51.50 \%$ compared with that of CK at the harvest stage, respectively (Fig. 1b). Summing up

227 the above, the samples of S2 and S3 were selected for RNA-seq analysis.

228

229 Effects of light intensity on anthocyanin composition in grape berry skin

230 UHPLC-MS/MS was used to detect anthocyanin composition in the berry skin at three

231 different developmental stages, and a total of 24 anthocyanin components were obtained (Fig.

232 2a, b and c). Among them, these 24 anthocyanins were present in both CK and A at the S3, and

233 only Cyanidin-3-O-coumaroylglucoside (trans) was not detected in B at S2 and S3 but

234 synthesized at $\mathrm{S} 1$. In addition, Cyanidin-3-O-coumaroylglucoside (cis) and

235 Dephinidin-3-O-coumaroylglucoside (cis) were not detected in $\mathrm{C}$ at $\mathrm{S} 3$ while

236 Dephinidin-3-O-coumaroylglucoside (cis) was detected at S1. Furthermore,

237 Cyanidin-3-O-coumaroylglucoside (cis), Peonidin-3- $O$-coumaroylglucoside (trans) and

238 Malvidin-3-O-coumaroylglucoside (trans) were not detected in D at S1, S2 and S3, whereas

239 the concentration of Cyanidin-3-O-coumaroylglucoside (cis) was very low in $\mathrm{CK}$ and other

240 bagging treatments from three development stages $\left(0.5-11.5 \mathrm{mg} \cdot \mathrm{kg}^{-1}\right)$. Therefore, these

241 results initially indicated that Peonidin-3-O-coumaroylglucoside (trans) and

242 Malvidin-3-O-coumaroylglucoside (trans) may be induced by light intensity, and also

243 suggested that the composition of anthocyanins decreased as the light intensity was

244 decreased.

246 Correlation and proportion analysis of individual anthocyanins and total anthocyanins

247 in the berry skin

248 Anthocyanin concentrations from the harvest stage were chosen for subsequent analysis since

249 all the bagged fruits were completely colored during this period. The concentration of

250 Peonidin-3-O-coumaroylglucoside (trans), Petunidin-3- $O$-coumaroylglucoside (cis) and 


\section{Manuscript to be reviewed}

251 Malvidin-3-O-coumaroylglucoside (trans) positively correlated $(r=0.775-0.892)$ with the total

252 anthocyanin concentration after bagging treatments (Suppl. Dataset S1). However, the

253 proportions of Peonidin-3-O-coumaroylglucoside (trans) and

254 Petunidin-3-O-coumaroylglucoside (cis) in $\mathrm{CK}$ were $1 \%$ and 0 , respectively (Fig. 3a).

255 Similarly, they were 0 in D (Fig. 3b). The proportion of Malvidin-3-O-coumaroylglucoside

256 (trans) was $12 \%$ in $\mathrm{CK}$ and was 0 in D. These results further indicated that

257 Malvidin-3-O-coumaroylglucoside (trans) was induced by light intensity. Nevertheless, the

258 proportion of Malvidin-3-O-coumaroylglucoside (cis) was $14 \%$ in D and $1 \%$ in $\mathrm{CK}$. The

259 concentration of Malvidin-3-O-acetylglucoside was highest in both $\mathrm{CK}$ and $\mathrm{D}$, which were

$26027 \%$ and $34 \%$, respectively. Therefore, except for light-inducible anthocyanin

261 Malvidin-3-O-coumaroylglucoside (trans), other anthocyanins which were both synthesized

262 in CK and D were considered to be light-independent anthocyanins, especially

263 Malvidin-3-O-coumaroylglucoside (cis) and Malvidin-3-O-acetylglucoside.

265 Comparative transcriptome analysis identified key processes and genes responsible for 266 anthocyanin accumulation regulated by light intensity

267 In order to further elucidate the molecular basis for anthocyanin accumulation, gene expression profiles in different light intensities were analyzed by comparative transcriptomic sequencing. After the sequencing quality control, $5.96 \mathrm{~Gb}$ clean bases were generated from the

270 ten libraries, and the Q30 base percentage of each sample was not less than $90.35 \%$ (Table S2).

271 Moreover, $97.77 \%-99.19 \%$ of high-quality $150 \mathrm{bp}$ reads were selected for further analysis

272 (Table S3). Clean reads of the various samples were aligned against the specified grape

273 reference genome. Mapped reads ranged from $46.36 \%$ to $78.43 \%$. Uniquely mapped reads and 274 multiple- mapped reads were ranged from $46.10 \%$ to $78.00 \%$ and $0.26 \%$ to $0.43 \%$, 275 respectively. 
276 Then, changes in gene expression levels were determined by comparing each of the

277 treatment with the $\mathrm{CK}$ at $\mathrm{S} 2$ and $\mathrm{S} 3$, which was $\mathrm{CK} 1$ versus $\mathrm{A} 1, \mathrm{CK} 1$ versus $\mathrm{B} 1, \mathrm{CK} 1$ versus

$278 \mathrm{C} 1, \mathrm{CK} 1$ versus D1 and CK2 versus A2, CK2 versus B2, CK2 versus C2, CK2 versus D2, 279 respectively. At S2, the down-regulated genes increased from 502 to 1,482, and the

280 up-regulated genes increased from 721 to 2,308 with the decrease of light intensity compared 281 with CK. At S3, the down-regulated genes increased from 1,592 to 2,429, whiles the 282 up-regulated genes increased from 1,326 to 1,599 with decreases in light intensity compared 283 with CK (Fig. S1). GO and KEGG enrichment analyses were conducted to understand the 284 functions of DEGs using all reference genes as background. GO term enrichment analysis 285 categorized the annotated sequences into three main categories: biological process, cellular 286 component and molecular function (Table S4). In the biological process category, the terms 287 "response to biotic stimulus" and "response to endogenous stimulus" was shared in CK1 versus B1, CK1 versus C1, CK1 versus D1, CK2 versus A2, CK2 versus B2, CK2 versus C2 and CK2 versus D2, except for in CK1 versus A1. The terms "response to stress" were shared in CK1

290 versus A1, CK1 versus B1, CK1 versus C1, CK1 versus D1, CK2 versus A2, CK2 versus B2, 291 CK2 versus C2 and CK2 versus D2. The terms "signal transduction", "regulation of cellular 292 process" and "cellular response to stimulus" were shared in CK1 versus B1, CK1 versus C1, 293 CK1 versus D1, CK2 versus A2, CK2 versus B2 and CK2 versus D2. In the cellular component 294 category, the terms "external encapsulating structure", "cell wall", "membrane" and "cell 295 periphery" were shared in CK1 versus A1, CK1 versus B1, CK1 versus C1, CK1 versus D1, 296 CK2 versus A2, CK2 versus B2, CK2 versus C2 and CK2 versus D2. In the molecular function 297 category, the DEGs were further classified into 13 major groups in CK2 versus B2 and CK2 versus $\mathrm{C} 2,11$ in $\mathrm{CK} 2$ versus D2, 10 in CK1 versus D1, 9 in CK1 versus B1 and CK2 versus A2, 2996 in CK1 versus A1, 4 in CK1 versus C1. Furthermore, KEGG pathways, including flavonoid 300 biosynthesis, circadian rhythm-plant, stilbenoid, diarylheptanoid and gingerol biosynthesis and 


\section{Manuscript to be reviewed}

301 phenylalanine metabolism were significantly enriched in CK1 versus A1, CK1 versus B1, CK1

302 versus $\mathrm{C} 1, \mathrm{CK} 1$ versus D1, CK2 versus $\mathrm{A} 2, \mathrm{CK} 2$ versus $\mathrm{B} 2, \mathrm{CK} 2$ versus $\mathrm{C} 2$ and $\mathrm{CK} 2$ versus

303 D2 (Table 1). Among these, flavonoid biosynthesis pathway was selected for subsequent

304 analysis.

305

306 Genes involved in flavonoid biosynthesis pathway

307 The expression levels of anthocyanin biosynthesis related genes were significantly different

308 among the different treatments. The expression levels of phenylalanine ammonium lyases

309 (PALs; VIT_06s0004g02620, VIT_13s0019g04460, VIT_16s0039g01360,

310 VIT_00s2849g00010, VIT_16s0039g01280 and VIT_08s0040g01710), trans-cinnamate

311 4-monooxygenase-like (C4H; VIT_06s0004g08150), CHS (VIT_16s0022g01190,

312 VIT_16s0022g01140, VIT_14s0068g00920, VIT_14s0068g00930 and VIT_05s0136g00260),

313 chalcone isomerase (CHI; VIT_13s0067g03820), F3'5'Hs/F3'Hs (VIT_06s0009g02970,

314 VIT_06s0009g02840 and VIT_06s0009g02860, VIT_17s0000g07210), flavanone

315 3-hydroxylase (F3H; VIT_04s0023g03370), DFR (VIT_18s0001g12800) and LDOX

316 (VIT_02s0025g04720) in D were significantly lower than that of CK at S2. The expression

317 levels of $F 3^{\prime} 5^{\prime} H s / F 3^{\prime} H$ s (VIT_06s0009g02970, VIT_06s0009g02840 and

318 VIT_17s0000g07210), F3H (VIT_04s0023g03370), and LDOX (VIT_02s0025g04720) were

319 significantly up-regulated compared with CK at S3. At S3, the expression levels of PALs

320 (VIT_13s0019g04460, VIT_16s0039g01360, VIT_00s2849g00010, VIT_16s0039g01280,

321 VIT_16s0039g01240, VIT_16s0039g01300 and VIT_08s0040g01710) and C4H

322 (VIT_06s0004g08150) in A2, B2 and D2 were down-regulated compared with that of CK2

323 (Fig. 4).

324

325 Regulation of MYB transcription factors by different light intensities

PeerJ reviewing PDF | (2018:11:32873:1:1:NEW 23 Jan 2019) 
326 At S3, MYB1R1 (VIT_00s0299g00060), MYB4B (VIT_04s0023g03710), MYB15

327

328

329

330

331

332

333

334

335

336

337

338

339

340

341

342

343

344

(VIT_05s0049g01020), MYBB1R1 (VIT_17s0000g07510), MYB44 (VIT_18s0001g09850), MYB80 (VIT_19s0015g01280) and MYB-like protein H isoform X1 (XLOC_013017) were up-regulated in bagged berry skin, of which MYB4B (VIT_04s0023g03710) and MYB15 (VIT_05s0049g01020) were most up-regulated in D treatment. However, MYB5B (VIT_06s0004g00570), MYB108-like protein 2 (VIT_07s0005g01950), MYBCS1 (VIT_08s0007g07230), MYB60 (VIT_08s0056g00800) and PHL6 (VIT_09s0054g01620) were significantly down-regulated (Fig. 5a).

The expression of $b H L H 47$ (VIT_14s0108g00480), MYC2 (VIT_15s0046g00320), bHLH137-like (VIT_17s0000g00430), bHLH79 (VIT_17s0000g05370), bHLH93 (VIT_18s0001g08040) and bHLH123 (VIT_19s0014g04670) in bagging treatments were significantly inhibited compared with that of CK at S3. bHLH147 (VIT_05s0077g00750), MYC1 (VIT_07s0104g00090), bHLH106(VIT_08s0040g01240), bHLH68 (VIT_09s0002g04120, VIT_11s0016g03560), bHLH144 (VIT_10s0003g02940), bHLH41 (VIT_11s0016g02070), bHLH30 (VIT_12s0028g02350), bHLH36 (VIT_12s0028g03550) and bHLH48 (VIT_08s0007g07870) were up-regulated in bagging treatments (Fig. 5b).

\section{Light-inducible and light-independent anthocyanins regulated by key candidate genes}

The concentration of light-inducible anthocyanin Malvidin-3-O-coumaroylglucoside (trans) was selected for the correlation analysis with genes involved in flavonoid biosynthesis pathway. In addition, although Malvidin-3- $O$-acetylglucoside accounted for the largest proportion of anthocyanin synthesis, Malvidin-3-O-coumaroylglucoside (cis) had the largest difference between $\mathrm{CK}$ and D. The reason was that Malvidin-3-O-coumaroylglucoside (cis) was an isomer of Malvidin-3-O-coumaroylglucoside (trans) and was therefore selected for correlation analysis. The concentration of Malvidin-3-O-coumaroylglucoside (trans) 
351 positively correlated with the expression of PALs (VIT_16s0039g01360,

352 VIT_00s2849g00010, VIT_16s0039g01240, VIT_08s0040g01710, VIT_16s0039g01300;

$353 r=0.773-0.795)$ and $F 3 H$ (VIT_18s0001g14310; $r=0.887)$, but it negatively correlated with

354 CHS (VIT_14s0068g00920), F3H (VIT_04s0023g03370) and F3'5'H (VIT_06s0009g02970)

355 ( $r=-0.973--0.796)$ (Suppl. Dataset S2). The concentration of

356 Malvidin-3-O-coumaroylglucoside (cis) positively correlated with the expression level of

$357 \quad F 3^{\prime} 5^{\prime} H$ (VIT_06s0009g02840; $r=0.923$ ) but it negatively correlated with $C H S$

358 (VIT_05s0136g00260; $r=-0.749$ ).

359 For transcription factors, light-inducible anthocyanin synthesis positively correlated with

360 MYB members (VIT_01s0011g04760, MYBC2-L1; VIT_02s0033g00390, MYBA2;

361

VIT_02s0033g00410, MYBA1; VIT_02s0033g00450, MYBA3; VIT_17s0000g06190,

362

MYB30; $r=0.702-0.812$ ) and bHLH (VIT_03s0038g01790, bHLH121; VIT_12s0028g02350,

bHLH30; VIT_17s0000g05370, bHLH79; $r=0.706-0.868)$, while negatively correlated with

MYB (VIT_04s0008g03720, Target of Myb protein 1; VIT_10s0116g00500, ETC1;

365

VIT_14s0006g01620, MYBC2; VIT_14s0066g01090, MYB24; $r=-0.844--0.709)$ and bHLH

366 (VIT_00s0274g00070, bHLH112; VIT_03s0038g04760, bHLH63; VIT_05s0029g00390,

bHLH77; VIT_05s0077g00750,bHLH147; VIT_08s0040g01240, bHLH106;

368

VIT_11s0016g03560, bHLH68; VIT_19s0014g04670, bHLH123; $r=-0.962--0.702)$ (Suppl.

369 Dataset S3). Light-independent anthocyanin synthesis was positively correlated with MYB

370 (VIT_16s0050g02530, TRY; r=0.882) and bHLH (VIT_05s0029g00390, bHLH77;

371 VIT_09s0002g04120, $b H L H 68 ; r=0.783-0.873)$, but it negatively correlated with other MYB members (VIT_06s0004g00570, MYB5B; VIT_07s0005g01950, MYB108-like protein 2;

373 VIT_09s0054g01620, PHL6; XLOC_013017, MYB-like protein H isoform X1;

$374 r=-0.939--0.700)$. 


\section{Manuscript to be reviewed}

375 To evaluate the validity of deep-sequencing data, 4 genes were selected for examination by

376

377

378

\section{Discussion}

380 qRT-PCR, of which only PAL (VIT_08s0040g01710) in A1 was not consistent with that of deep-sequencing, indicating the reliability of high-throughput data (Fig. 6).

\section{Complete darkness decreased the concentration and composition of total anthocyanins,} delayed the veraison, but did not affect the coloration of grape berry skin during harvest

Light is an important factor which affects fruit development and it also participates in a series of processes such as fruit morphological development, quality formation and changes in molecular structure (Feng et al., 2013; Takos et al., 2016). Fruit color is an important parameter for ripening and quality evaluation of fresh and processed fruit (De et al., 2017; Henwood et al., 2018). In the present research, the veraison of B was delayed for 3 days compared with $\mathrm{CK}$ whiles the veraison of $\mathrm{C}$ and $\mathrm{D}$ was delayed for 10 days. The composition of anthocyanins in berries affects the color tone and color stability of resulting wines (Rustioni et al., 2013). Also, the results of this study suggested that the components of anthocyanins decreased gradually with decreases in the light transmittance. All the 24 anthocyanin components detected were present in both $\mathrm{CK}$ and $\mathrm{A}$, while Cyanidin-3-O-coumaroylglucoside (cis) was not detected in $\mathrm{C}$, Cyanidin-3-O-coumaroylglucoside (cis), Peonidin-3-O-coumaroylglucoside (trans) and Malvidin-3-O-coumaroylglucoside (trans) were not detected in D from the three development stages. The effect of fruit exposure to light has been investigated in previous studies (Jutamanee et al., 2016; Llorente et al., 2016). As observed in apple (Honda et al., 2017; Honda \& Moriya, 2018) and pear (Feng et al., 2010), the bagged fruits displayed no color, but the pericarp turned red when exposed to sunlight. In the absence of light, the anthocyanin synthesis from berry skin of grape cv. 'Jingxiu' was inhibited, while the grape cv. 'Jingyan' 


\section{Manuscript to be reviewed}

400 remained unchanged (Zheng et al., 2009). Similarly, although different fruit bags significantly

401 reduced the concentrations of total anthocyanins in our research, the appearance of the berry

402 skin in bagged berries was not different from that of the $\mathrm{CK}$ at harvest stage, suggesting that

403 grape varieties respond differently to light (Cortell et al., 2006). In summary, complete

404 darkness decreased the concentration and composition of total anthocyanins, delayed the

405 veraison, but did not affect the coloration of the berry skin during harvest in grape cv.

406 'Marselan'.

407

408 Light-independent anthocyanin is dominant for berry skin coloration in grape cv.

409 'Marselan'

410 Fruit -zone shading reduced total anthocyanin accumulation and decreased the 3 '-hydroxylated

411 anthocyanin concentration but increased the $3^{\prime}, 5^{\prime}$-hydroxylated anthocyanin concentration in

412 'Nebbiolo' grapes (Price et al., 1995). Similarly, the accumulation of 3',4',5'-hydroxylated

413 anthocyanin in grape cv. 'Yan-73' decreased under dark conditions, while the concentration of

4143 3',4'-hydroxylated anthocyanin increased (Guan et al., 2014). Studies have shown that

415 trans-structured substances are more stable than cis-structured substances (Schieber \& Carle,

416 2005), and changes in the external environment usually alter the concentration of these

417 substances (Spanos \& Wrolstad, 1990; Versari et al., 2001). In grape cv. 'Redglobe', both

418 UV-C and refrigeration treatment increased the concentration of cis- and trans-piceid (Crupi

419 et al., 2013). The concentration of trans-resveratrol increased when the temperature

420 increased, while the concentration of cis-resveratrol decreased (Kolouchová-HanzlíKová et

421 al., 2004). In our work, Malvidin-3-O-coumaroylglucoside (trans), which showed a

422 significant positive correlation with the total concentration of anthocyanins at the harvest

423 stage and were not detected in D, were considered to be light-induced anthocyanin. These

424 was trans-structures with good stability. Other anthocyanins which were both synthesized in

PeerJ reviewing PDF | (2018:11:32873:1:1:NEW 23 Jan 2019) 
$425 \mathrm{CK}$ and D were considered to be light-independent anthocyanins. The cis-structures of

426 light-independent anthocyanins, Peonidin-3-O-coumaroylglucoside (cis) and

427 Malvidin-3-O-coumaroylglucoside (cis), were involved in light-independent anthocyanins.

428 Therefore, these results indicated that the intensity of light could change the isomerization

429 level of anthocyanins in grapes. In addition to the light-inducible anthocyanins which

430 accounted for $13 \%$ in CK, the proportion of light-independent anthocyanins accounted for

$43187 \%$ (Fig. 3a). Therefore, light-independent anthocyanins are the dominant for berry skin

432 coloration in grape cv. 'Marselan'.

434 The synthesis of light-inducible anthocyanins and light-independent anthocyanins may

435 be regulated by specific genes

436 Anthocyanin synthesis belongs to the flavonoid metabolic pathway, which involves a series of

437 metabolic reactions and intermediates synthesis, resulting in different components of

438 anthocyanins (Matsui et al., 2016). Shading and bagging experiments showed that light could

439 stimulate the up-regulation of anthocyanin synthesis related genes, thereby increasing

440 anthocyanin accumulation in fruits (Guan et al., 2016; Zhang et al., 2016). Studies have shown

441 that PAL gene expression is responsive to light (Liang et al., 1989; Huang et al., 2010; Zhu et

442 al., 2018). Similarly, the results of the present study revealed that the expression levels of

443 PALs (VIT_16s0039g01360, VIT_00s2849g00010, VIT_16s0039g01240,

444 VIT_08s0040g01710, VIT_16s0039g01300) and F3H(VIT_18s0001g14310) which

445 positively correlated with the concentration of Malvidin-3-O-coumaroylglucoside (trans),

446 were considered to positively regulate the synthesis of light-inducible anthocyanins. Zhang

447 and others also found that over-expression of the $C H S$ gene enhanced high light resistance by

448 synthesizing more anthocyanins in Arabidopsis leaves (Zhang et al., 2017). The expression of

449 CHS (VIT_05s0136g00260) which was negatively correlated with the concentration of

PeerJ reviewing PDF | (2018:11:32873:1:1:NEW 23 Jan 2019) 


\section{Manuscript to be reviewed}

450 Malvidin-3-O-coumaroylglucoside (cis) in the present study was considered to be related to

451 the synthesis of light-independent anthocyanins. The accumulation of $3^{\prime}, 4^{\prime}, 5^{\prime}$-hydroxylated

452 and $3^{\prime}, 4^{\prime}$-hydroxylated anthocyanins respond differently to dark conditions and was related to

453 the expression levels of $V v F 3^{\prime} H$ and $V v F 3^{\prime} 5^{\prime} H$ (Guan et al., 2014). Similarly, the expression

454 levels of $C H S$ (VIT_14s0068g00920), F3H (VIT_04s0023g03370) and F3'5'H

455 (VIT_06s0009g02970) which negatively correlated with the concentration of

456 Malvidin-3-O-coumaroylglucoside (trans) in our work, were considered to negatively

457 regulate the synthesis of light-inducible anthocyanins. In addition, the expression of $F 3^{\prime} 5^{\prime} H$

458 (VIT_06s0009g02840) which positively correlated with the concentration of

459 Malvidin-3-O-coumaroylglucoside (cis), was considered to positively regulate the synthesis

460 of light-independent anthocyanins.

461 Members of the MYB transcription factor families, particularly, MYBA1, MYBA2, MYB5A,

$462 M Y B 5 B, M Y B P A 1$ and $M Y B P A 2$, were involved in the regulation of structural genes in the

463 flavonoid pathway (Ali, 2011; Cavallini et al., 2014). The expression of R2R3-MYB

464 transcription factor $M Y B F 1$ was light-inducible, implicating $M Y B F 1$ in the transcripts

465 regulation of FLS in grape (Czemmel et al., 2009). MYB10 was most responsive to light while

466 the transcripts declined to undetectable levels in the fruit preserved in the dark (Daniela et

467 al., 2013). In this study, light-inducible anthocyanin synthesis positively correlated with

468 MYBC2-L1, MYBA2, MYBA1, MYBA3, MYB30, bHLH121, bHLH30 and bHLH79, but it

469 negatively correlated with target of MYB protein 10, ETC1, MYBC2, MYB24, bHLH112,

$470 \quad$ bHLH63, bHLH77, bHLH147, bHLH106, bHLH68 and bHLH123. Light-independent

471 anthocyanin synthesis was positively correlated with $T R Y, b H L H 77$ and $b H L H 68$, but it

472 negatively correlated with MYB5B, MYB108-like protein 2, PHL6 and MYB-like protein H

473 isoform $\mathrm{X} 1$.

474

PeerJ reviewing PDF | (2018:11:32873:1:1:NEW 23 Jan 2019) 


\section{Manuscript to be reviewed}

\section{Conclusion}

476 Light could affect the accumulation of light-inducible anthocyanins by positively regulating

477 the expression levels of PALs, F3H (VIT_18s0001g14310), MYBC2-L1, MYBA2, MYBA1,

478 MYBA3, MYB30, bHLH121, bHLH30 and bHLH79, while negatively regulating the

479 expression of $C H S$ (VIT_14s0068g00920), F3H(VIT_04s0023g03370), F3'5'H

480 (VIT_06s0009g02970), target of Myb protein 10, ETC1, MYBC2, MYB24, bHLH112,

481 bHLH63, bHLH77, bHLH147, bHLH106, bHLH68 and bHLH123. On the contrary, darkness

482 may promote the expression of $F^{\prime} 3^{\prime}{ }^{\prime} H$ (VIT_06s0009g02840), TRY, bHLH77 and bHLH68

483 but inhibit the expression of CHS (VIT_05s0136g00260), MYB5B, MYB108-like protein 2,

484 PHL6 and MYB-like protein $\mathrm{H}$ isoform X1, and therefore promote the synthesis of

485 light-independent anthocyanins.

486

487 Acknowledgements

488

489 References

490 Ali MB. 2011. Berry skin development in norton grape: distinct patterns of transcriptional

491 regulation and flavonoid biosynthesis. BMC Plant Biology 11(1): 7

492 Azuma A, Yakushiji H, Koshita Y, Kobayashi S. 2012. Flavonoid biosynthesis-related

493 genes in grape skin are differentially regulated by temperature and light conditions. Planta

494 236(4): 1067-1080

495 Ban Y, Honda C, Hatsuyama Y, Igarashi M, Bessho H, Moriguchi T. 2007. Isolation and

496 functional analysis of a MYB transcription factor gene that is a key regulator for the

497 development of red coloration in apple skin. Plant and Cell Physiology 48(7): 958-970. 


\section{Manuscript to be reviewed}

498 Bellincontro A, Fardelli A, Santis DD, Botondi R, Mencarelli F. 2010. Postharvest ethylene

499 and 1-MCP treatments both affect phenols, anthocyanins, and aromatic quality of Aleatico

$500 \quad$ grapes and wine. Australian Journal of Grape and Wine Research 12(2): 141-149

501 Carbone F, Preuss A, De Vos RC, D'Amico E, Perrotta G, Bovy AG, Martens G, Rosati C.

502 2009. Developmental, genetic and environmental factors affect the expression of flavonoid

503 genes, enzymes and metabolites in strawberry fruits. Plant Cell and Environment 32(8):

$504 \quad 1117-1131$

505 Castellarin SD, Gaspero GD. 2007. Transcriptional control of anthocyanin biosynthetic

506 genes in extreme phenotypes for berry pigmentation of naturally occurring grapevines.

507 BMC Plant Biology 7(1): 46

508 Cavallini E, Zenoni S, Finezzo L, Guzzo F, Zamboni A, Avesani L, Tornielli GB. 2014.

509 Functional diversification of grapevine MYB5a and MYB5b in the control of flavonoid

510 biosynthesis in a petunia anthocyanin regulatory mutant. Plant and Cell Physiology 55(3):

$511 \quad 517-534$

512 Cheng G, Zhou SH, Huang Y, Zhang Y, Guan JX, Yang Y, Wen RD, Zhang J. 2017.

513 Analysis of anthocyanin composition characteristics and gene expression patterns of 'Guipu

$514 \quad$ No.6’ grape. Plant Physiology Journal 53(1): 103-114

515 Cominelli E, Gusmaroli G, Allegra D, Galbiati M, Wade HK, Jenkins GI, Tonelli C. 2008.

516 Expression analysis of anthocyanin regulatory genes in response to different light qualities

517 in Arabidopsis thaliana. Journal of Plant Physiology 165(8): 886-894

518 Cortell JM, Kennedy JA. 2006. Effect of shading on accumulation of flavonoid compounds

519 in (Vitis vinifera L.) pinot noir fruit and extraction in a model system. Journal of

$520 \quad$ Agricultural and Food Chemistry 54(22): 8510-8520

PeerJ reviewing PDF | (2018:11:32873:1:1:NEW 23 Jan 2019) 


\section{Manuscript to be reviewed}

521 Crupi P, Pichierri A, Basile T, Antonacci D. 2013. Postharvest stilbenes and flavonoids

522

523

524

525

526

527

528

529

530

531

532

533

534

535

536

537

538

539

540

541

542

543

enrichment of table grape cv redglobe (Vitis vinifera L.) as affected by interactive UV-C exposure and storage conditions. Food Chemistry 141(2): 802-808

\section{Czemmel S, Stracke R, Weisshaar B, Cordon N, Harris NN, Walker AR, Robinson SP,} Bogs J. 2009. The grapevine R2R3-MYB transcription factor $V v M Y B F 1$ regulates flavonol synthesis in developing grape berries. Plant Physiology 151(3): 1513-1530

Daniela R, Espley RV, Henry-Kirk RA, Carlo A, Vanina Z, Hellens RP, Costa G, Allan AC. 2013. Transcriptional regulation of flavonoid biosynthesis in nectarine (prunus persica) by a set of R2R3 MYB transcription factors. BMC Plant Biology 13(1): 68-68

De RI, Ponce MT, Malovini E, Deis L, Cavagnaro B, Cavagnaro P. 2017. Loss of anthocyanins and modification of the anthocyanin profiles in grape berries of malbec and bonarda grown under high temperature conditions. Plant Science 258: 137-145

Doostdar H, Shapiro JP, Niedz R, Burke MD, McCollum TG, McDonald RE, Mayer RT. 1995. A cytochrome $\mathrm{P} 450$ mediated naringenin 3 '-hydroxylase from sweet orange cell cultures. Plant and Cell Physiology 36(1): 69-77

Downey MO, Harvey JS, Robinson SP. 2010. The effect of bunch shading on berry development and flavonoid accumulation in shiraz grapes. Australian Journal of Grape and Wine Research 10(1): 55-73

Feng F, Li M, Ma F, Cheng L. 2013. Phenylpropanoid metabolites and expression of key genes involved in anthocyanin biosynthesis in the shaded peel of apple fruit in response to sun exposure. Plant Physiology and Biochemistry 69: 54-61

Feng S, Wang Y, Yang S, Xu Y, Chen X. 2010. Anthocyanin biosynthesis in pears is regulated by a R2R3-MYB transcription factor PyMYB10. Planta 232(1): 245-255 
544 Figueiredo-González M, Cancho-Grande B, Boso S, Santiago JL, Martínez MC,

545 Simal-Gándara J. 2013. Evolution of flavonoids in Mouratón berries taken from both bunch

546 halves. Food Chemistry 138(2-3): 1868-1877

547 Figueiredo-González M, Martínez-Carballo E, Cancho-Grande B, Santiago JL,

548 Martínez MC, Simal-Gándara J. 2012a. Pattern recognition of three Vitis vinifera L. red

549 grapes varieties based on anthocyanin and flavonol profiles, with correlations between their

550 biosynthesis pathways. Food Chemistry 130(1): 9-19

551 Figueiredo-González M, Simal-Gándara J, Boso S, Martínez MC, Santiago JL,

552 Cancho-Grande B. 2012b. Anthocyanins and flavonols berries from Vitis vinifera L. cv.

553 Brancellao separately collected from two different positions within the cluster. Food

554 Chemistry 135(1): 47-56

555 Figueiredo-González M, Simal-Gándara J, Boso S, Martínez MC, Santiago JL,

556 Cancho-Grande B. 2012c. Flavonoids in Gran Negro berries collected from shoulders and

557 tips within the cluster, and comparison with Brancellao and Mouratón varieties. Food

558 Chemistry 133(3): 806-815

559 Fukui Y, Tanaka Y, Kusumi T, Iwashita T, Nomoto K. 2003. A rationale for the shift in

560 colour towards blue in transgenic carnation flowers expressing the flavonoid

$561 \quad 3 ', 5$ '-hydroxylase gene. Phytochemistry 63(1): 15-23

562 Guan L, Li JH, Fan PG, Hua S, Fang JB, Dai ZW, Delrot S, Wang LC, Wang LJ, Wu BH.

563 2014. Regulation of anthocyanin biosynthesis in tissues of a teinturier grape cultivar under

$564 \quad$ sunlight exclusion. American Journal of Enology and Viticulture 65: in press

565 He B, Zhang LL, Yue XY, Liang J, Jiang J, Gao XL, Yue PX. 2016. Optimization of

566 Ultrasound-Assisted extraction of phenolic compounds and anthocyanins from blueberry

567 (Vaccinium ashei) wine pomace. Food Chemistry 204: 70-76

PeerJ reviewing PDF | (2018:11:32873:1:1:NEW 23 Jan 2019) 


\section{Manuscript to be reviewed}

568 Henwood RJT, Wargent JJ, Black M, Heyes JA. 2018. Environmental and management

569 factors contributing to variability in flesh colour of a red kiwifruit cultivar in New Zealand.

$570 \quad$ Scientia Horticulturae 235: 21-31

571 Honda C, Iwanami H, Naramoto K, Maejima T, Kanamaru K, Moriya-Tanaka Y,

572 Hanada T, Wada M. 2017. Thinning and bagging treatments and the growing region

573 influence anthocyanin accumulation in red-fleshed apple fruit. Horticulture Journal 86(3):

$574 \quad 291-299$

575 Honda C, Moriya S. 2018. Anthocyanin biosynthesis in apple fruit. Horticulture Journal

$576 \quad$ OKD-R01

577 Huang J, Gu M, Lai Z, Fan B, Shi K, Zhou YH, Yu J, Chen Z. 2010. Functional analysis of

578 the Arabidopsis PAL gene family in plant growth, development and response to

579 environmental stress. Plant Physiology pp-110

580 Ilieva F, Veličkovska SK, Dimovska V, Spasov H. 2016. The impact of some wine-making

581 practices on the quality of Vranec red wines from Macedonia produced by the

582 newly-selected local strain "F-78". Food Chemistry 194: 1123-1131

583 Jia H J, Araki A, Okamoto G. 2005. Influence of fruit bagging on aroma volatiles and skin

584 coloration of 'Hakuho'peach (Prunus persica Batsch). Postharvest Biology and Technology

$585 \quad 35(1): 61-68$

586 Jutamanee K, Onnom S. 2016. Improving photosynthetic performance and some fruit quality

$587 \quad$ traits in mango trees by shading. Photosynthetica 54(4): $1-9$

588 Kanehisa M, Goto S, Kawashima S, Okuno Y, Hattori M. 2004. The KEGG resource for

589 deciphering the genome. Nucleic Acids Research 2(suppl_1): D277-D280.

590 Kolouchová-HanzlíKová I, Melzoch K, Filip V, Šmidrkal J. 2004. Rapid method for

591 resveratrol determination by HPLC with electrochemical and UV detections in wines. Food

$592 \quad$ Chemistry 87(1): 151-158

PeerJ reviewing PDF | (2018:11:32873:1:1:NEW 23 Jan 2019) 
593 Kondo S, Tomiyama H, Rodyoung A, Okawa K, Ohara H, Sugaya S, Norihiko T,

594 Nobuhiro H. 2014. Abscisic acid metabolism and anthocyanin synthesis in grape skin are

595 affected by light emitting diode (led) irradiation at night. Journal of Plant Physiology

596 171(10): 823-829

597 Lepiniec L, Debeaujon I, Routaboul JM, Baudry A, Pourcel L, Nesi N, Caboche M.

598 2006. Genetics and biochemistry of seed flavonoids. Annual Review of Plant Biology 57(1):

$599 \quad 405-430$

600 Li YY, Mao K, Zhao C, Zhao XY, Zhang HL, Shu HR, Hao YJ. (2012). MdCOP1

601 ubiquitin E3 ligases interact with MdMYB1 to regulate light-induced anthocyanin

602 biosynthesis and red fruit coloration in apple. Plant Physiology: pp-112.

603 Liang NN, Zhu BQ, Han S, Wang JH, Pan QH, Reeves MJ, Duan CQ, He F. 2014.

604 Regional characteristics of anthocyanin and flavonol compounds from grapes of four Vitis

605 vinifera varieties in five wine regions of China. Food Research International 64: 264-274

606 Liang XW, Dron M, Cramer CA, Dixon RA, Lamb CJ. 1989. Differential regulation of

607 phenylalanine ammonia-lyase genes during plant development and by environmental cues.

$608 \quad$ Journal of Biological Chemistry 264: 14486-14492

609 Lin-Wang K, Bolitho K, Grafton K, Kortstee A, Karunairetnam S, McGhie TK, Espley

610 RV, Hellens RP, Allan AC. 2010. An R2R3 MYB transcription factor associated with

611 regulation of the anthocyanin biosynthetic pathway in Rosaceae. BMC Plant Biology 10(1):

$612 \quad 50$

613 Liu Y, Linwang K, Deng C, Warran B, Wang L, Yu B, Yang HY, Wang J, Espley RV,

614 Zhang JL, Wang D, Allan AC. 2015. Comparative transcriptome analysis of white and

615 purple potato to identify genes involved in anthocyanin biosynthesis. PLoS One 10:

$616 \quad \mathrm{e} 0129148$

PeerJ reviewing PDF | (2018:11:32873:1:1:NEW 23 Jan 2019) 


\section{Manuscript to be reviewed}

617 Livak KJ, Schmittgen TD. 2001. Analysis of relative gene expression data using real-time quantitative PCR and the $2^{-\Delta \Delta \mathrm{CT}}$ method. Methods 25: 402-408

Llorente B, D'Andrea L, Ruiz-Sola MA, Botterweg E, Pulido P, Andilla J, Alvarez PL,

Concepcion MR. 2016. Tomato fruit carotenoid biosynthesis is adjusted to actual ripening progression by a light-dependent mechanism. Plant Journal 85: 107-119

622 Lopes-da-Silva F, de Pascual-Teresa S, Rivas-Gonzalo J, Santos-Buelga C. 2002. Identification of anthocyanin pigments in strawberry (cv Camarosa) by LC using DAD and ESI-MS detection. European Food Research and Technology 214: 248-253

Maier A, Hoecker U. 2015. COP1/SPA ubiquitin ligase complexes repress anthocyanin accumulation under low light and high light conditions. Plant Signal \& Behavior 10(1): e970440

Mao J, Li W, Mi B, Ma Z, Dawuda MM, Zuo C, Zhang Y, Jiang X, Chen B. 2018. Transcriptome analysis revealed glucose application affects plant hormone signal transduction pathway in "Red Globe" grape plantlets. Plant Growth Regulation 84(7):

$631 \quad 45-56$

632 Matsui K, Hisano T, Yasui Y, Mori M, Walker AR, Morishita T, Katsu K. 2016. Isolation 633 and characterization of genes encoding leucoanthocyanidin reductase $($ FeLAR) and 634 anthocyanidin reductase (FeANR) in buckwheat (Fagopyrum esculentum). Journal of Plant 635 Physiology 205: 41-47

636 Miao L, Zhang Y, Yang X, Xiao J, Zhang H, Zhang Z, Wang Y, Jiang G. 2016. Colored 637 light-quality selective plastic films affect anthocyanin content, enzyme activities, and the 638 expression of flavonoid genes in strawberry (Fragariaxananassa) fruit. Food Chemistry 639 207: 93-100 


\section{Manuscript to be reviewed}

640 Moro L, Hassimotto NM, Purgatto E. 2017. Postharvest auxin and methyl jasmonate effect

641 on anthocyanin biosynthesis in red raspberry (Rubus idaeus L.). Journal of Plant Growth

642 Regulation 1: 1-10

643 Ortega-Regules A, Romero-Cascales I, López-Roca J., Ros-García JM, Gómez-Plaza E.

644 2006. Anthocyanin fingerprint of grapes: environmental and genetic variations. Journal of

645 the Science of Food and Agriculture 86(10): 1460-1467

646 Pérez-Gregorio MR, Regueiro J, Simal-Gándara J, Rodrigues AS, Almeida DPF. 2014.

647 Increasing the added-value of onions as a source of antioxidant flavonoids: a critical review.

$648 \quad$ Critical Reviews in Food Science and Nutrition 54(8): 1050-1062

649 Perez-Gregorio R, Simal-Gandara J. 2017a. A critical review of the characterization of

650 polyphenol-protein interactions and of their potential use for improving food quality.

$651 \quad$ Current Pharmaceutical Design 23(19): 2742-2753

652 Perez-Gregorio R, Simal-Gandara J. 2017b. A Critical review of bioactive Food

653 Components, and of their functional mechanisms, biological effects and health outcomes.

654 Current Pharmaceutical Design 23(19): 2731-2741

655 Price SF, Breen PJ, Valladao M, Watson BT. 1995. Cluster sun exposure and quercetin in 656 Pinot noir grapes and wine. American Journal of Enology and Viticulture 46(2): 187-194

657 Quijada-Morín N, Regueiro J, Simal-Gándara J, Tomás E, Rivas-Gonzalo JC,

658 Escribano-Bailón MT. 2012. Relationship between the sensory-determined astringency

659 and the flavanolic composition of red wines. Journal of Agricultural and Food Chemistry

$660 \quad$ 60(50): $12355-12361$

661 Rabino I, Mancinelli AL. 1986. Light, temperature and anthocyanin production. Plant

$662 \quad$ Physiology 81(3): 922-924

663 Regueiro J, Sánchez-González C, Vallverdú-Queralt A, Simal-Gándara J,

664 Lamuela-Raventós R, Izquierdo-Pulido M. 2014. Comprehensive identification of walnut 


\section{Manuscript to be reviewed}

665 polyphenols by liquid chromatography coupled to linear ion trap-Orbitrap mass

666 spectrometry. Food Chemistry 152: 340-348

667 Rustioni L, Di Meo F, Guillaume M, Failla O, Trouillas P. 2013. Tuning color variation in

668 grape anthocyanins at the molecular scale. Food Chemistry 141(4): 4349-4357

669 Schieber A, Carle R. 2005. Occurrence of carotenoid cis - isomers in food: technological,

670 analytical, and nutritional implications. Trends in Food Science \& Technology 16(9):

$671 \quad 416-422$

672 Spanos GA, Wrolstad RE. 1990. Influence of processing and storage on the phenolic

673 composition of Thompson seedless grape juice. Journal of Agricultural and Food Chemistry

$674 \quad 38(7): 1565-1571$

675 Takos AM, Jaffe FW, Jacob SR, Bogs J, Robinson SP, Walker AR. 2006. Light-inducible

676 expression of a MYB gene regulates anthocyanin biosynthesis in red apples. Plant

677 Physiology 142(3): 1216-1232

678 Takos AM, Jaffé FW, Jacob SR, Bogs J, Robinson SP, Walker AR. 2006. Light-induced

679 expression of a MYB gene regulates anthocyanin biosynthesis in red apples. Plant

$680 \quad$ Physiology 142(3): 1216-1232.

681 Tao R, Bai S, Ni J, Yang Q, Zhao Y, Teng Y. 2018. The blue light signal transduction

682 pathway is involved in anthocyanin accumulation in 'Red Zaosu' pear. Planta 248: 37-48

683 Versari A, Parpinello GP, Tornielli GB, Ferrarini R, Giulivo C. 2001. Stilbene compounds

684 and stilbene synthase expression during ripening, wilting, and UV treatment in grape cv.

685 Corvina. Journal of Agricultural and Food Chemistry 49(11): 5531-5536

686 Wang X, Wei Z, Ma F. 2015. The effects of fruit bagging on levels of phenolic compounds

687 and expression by anthocyanin biosynthetic and regulatory genes in red-fleshed apples.

688 Process Biochemistry 50(11): 1774-1782

PeerJ reviewing PDF | (2018:11:32873:1:1:NEW 23 Jan 2019) 


\section{Manuscript to be reviewed}

689 Xie C, Mao X, Huang J, Ding Y, Wu J, Dong S, Kong L, Gao G, Li CY, Wei L. 2011.

690 KOBAS 2.0: a web server for annotation and identification of enriched pathways and

691 diseases. Nucleic Acids Research 39 (suppl 2): 316-322

692 Xu F, Cao S, Shi L, Chen W, Su X, Yang Z. 2014. Blue light irradiation affects anthocyanin

693 content and enzyme activities involved in postharvest strawberry fruit. Journal of

$694 \quad$ Agricultural and Food Chemistry 62(20): 4778-4783

695 Zhang HN, Li WC, Wang HC, Shi SY, Shu B, Liu LQ, Wei YZ, Xie JH. 2016.

696 Transcriptome profiling of light-regulated anthocyanin biosynthesis in the pericarp of litchi.

$697 \quad$ Frontiers in Plant Science 7: 963

698 Zhang XH, Zheng XT, Sun BY, Peng CL, Chow WS. 2017. Over-expression of the CHS

699 gene enhances resistance of Arabidopsis leaves to high light. Environmental and

$700 \quad$ Experimental Botany 154: 33-43

701

Zhang Y, Jiang L, Li Y, Chen Q, Ye Y, Zhang Y, Luo Y, Sun B, Wang X, Tang H. 2018.

702 Effect of red and blue light on anthocyanin accumulation and differential gene expression in

703 strawberry (Fragaria $\times$ ananassa). Molecules 23(4): 820

704 Zhang Y, Xu S, Cheng Y, Peng Z, Han J. 2018. Transcriptome profiling of

705 anthocyanin-related genes reveals effects of light intensity on anthocyanin biosynthesis in

$706 \quad$ red leaf lettuce. PeerJ 6(4): e4607

707 Zheng Y, Li T, Liu H, Pan Q, Zhan J, Huang W. 2009. Sugars induce anthocyanin

708 accumulation and flavanone3-hydroxylase expression in grape berries. Plant Growth

$709 \quad$ Regulation 58(3): 251-260

710 Zhou TS, Zhou R, Yu YB, Xiao Y, Li DH, Xiao B, Yu O, Yang YJ. 2016. Cloning and

711 characterization of a flavonoid 3'-hydroxylase gene from tea plant (Camellia sinensis).

712 International Journal of Molecular Sciences 17(2): 261

PeerJ reviewing PDF | (2018:11:32873:1:1:NEW 23 Jan 2019) 
713 Zhu YF, Su J, Yao GF, Liu HN, Gu C, Qin MF, Bai B, Cai SS, Wang GM, Wang RZ, Shu

714 Q, Wu J. 2018. Different light-response patterns of coloration and related gene expression

715 in red pears (Pyrus L.). Scientia Horticulturae 229: 240-251

716 Zoratti L, Karppinen K, Luengo Escobar A, Häggman H, Jaakola L. 2014.

717 Light-controlled flavonoid biosynthesis in fruits. Frontiers in Plant Science 5: 534

718

719

$720 \quad$ Figure legends

721 Figure 1. Effects of different fruit bags on berry skin color. Bags with light transmittance of $72250 \%, 15 \%, 5 \%$ and 0 were performed at 45 days after flowering and designated as $\mathrm{CK}, \mathrm{A}, \mathrm{B}$,

$723 \mathrm{C}$ and D, respectively. Unbagged berries as a control. a Close-up views of wine grape cv.

724 'Marselan' berry fruit from different bagging treatments at S1, S2 and S3. b Changes of

725 anthocyanin concentration at the three development stages. Error bars represent the \pm SE of

726 three biological replicates, and the asterisks represent significant differences from the control,

727 with $P<0.05$ (*) or $P<0.01(* *)$

728

729 Figure 2. Heatmap of the concentration of anthocyanins in different bagging treatments at S1,

730 S2 and S3. Colors indicate the concentration of the anthocyanin composition. a Composition

731 and concentration of anthocyanins in S1. b Composition and concentration of anthocyanins in

732 S2. c Composition and concentration of anthocyanins in S3

733

734 Figure 3. The proportion of individual anthocyanins in total anthocyanin concentration from

$735 \mathrm{CK}($ panel $\mathbf{a})$ and $\mathrm{D}($ panel $\mathbf{b})$ treatments at S3

736 
737 Figure 4. Heatmap of expressed genes assigned to anthocyanins synthesis in different bagging

738 treatments. Colors indicate expression values of the genes. Expression values of ten libraries

739 are presented as FPKM normalized $\log 2$ transformed counts

740

741 Figure 5. Heatmap of MYB and basichelix-loop-helix (bHLH) transcription factors in

742 different bagging treatments. Colors indicate expression values of the genes. Expression values

743 of ten libraries are presented as FPKM normalized $\log 2$ transformed counts

744

745 Figure 6. qRT-PCR validation of 4 candidate genes related to anthocyanins synthesis from the

746 different bagging treatments. The left y-axis indicates relative gene expression levels were

747 determined by qRT-PCR and analyzed using $2^{-\Delta \Delta C T}$ Method. The $\mathrm{x}$-axis indicates different

748 treatments. All qRT-PCR for each gene used three biological replicates, with three technical

749 replicates per experiments. Error bars indicate \pm SE, and the asterisks represent significant

750 differences from the control, with $P<0.05(*)$ or $P<0.01(* *)$.

751

752 Figure S1. Venn diagram of numbers of DEGs in different bagging treatments: CK1 versus A1,

753 CK1 versus B1, CK1 versus $\mathrm{C} 1$ and CK1 versus D1 (S2), CK2 versus A2, CK2 versus B2,

754 CK2 versus C2 and CK2 versus D2 (S3) 


\section{Table 1 (on next page)}

Significantly enriched pathways of DEGs between different treatments 


\section{Table 1 Significantly enriched pathways of DEGs between different treatments}

\begin{tabular}{|c|c|c|c|c|}
\hline Pathway ID & Pathway & $\begin{array}{l}\text { Number } \\
\text { DEGs }\end{array}$ & $\begin{array}{c}\text { of Background } \\
\text { number }\end{array}$ & $\begin{array}{l}Q \text {-value } \\
(<0.01)\end{array}$ \\
\hline & CK1-vs-A1 & & & \\
\hline ko00941 & Flavonoid biosynthesis & 39 & 73 & 0.000000 \\
\hline ko04712 & Circadian rhythm - plant & 28 & 60 & 0.000000 \\
\hline ko00945 & Stilbenoid, diarylheptanoid and gingerol biosynthesis & 13 & 23 & 0.000000 \\
\hline ko00360 & Phenylalanine metabolism & 19 & 57 & 0.000000 \\
\hline \multirow[t]{2}{*}{ ko00940 } & Phenylpropanoid biosynthesis & 22 & 172 & 0.002593 \\
\hline & CK1-vs-B1 & & & \\
\hline ko00196 & Photosynthesis - antenna proteins & 11 & 19 & 0.000008 \\
\hline ko04626 & Plant-pathogen interaction & 34 & 171 & 0.000138 \\
\hline ko00941 & Flavonoid biosynthesis & 17 & 73 & 0.004055 \\
\hline ko00073 & Cutin, suberine and wax biosynthesis & 9 & 25 & 0.004055 \\
\hline \multirow[t]{2}{*}{ ko04075 } & Plant hormone signal transduction & 34 & 214 & 0.007062 \\
\hline & CK1 versus $\mathrm{C} 1$ & & & \\
\hline ko00941 & Flavonoid biosynthesis & 18 & 73 & 0.000021 \\
\hline \multirow[t]{2}{*}{ ko04626 } & Plant-pathogen interaction & 27 & 171 & 0.000170 \\
\hline & CK1 versus D1 & & & \\
\hline ko00941 & Flavonoid biosynthesis & 52 & 73 & 0.000000 \\
\hline ko04712 & Circadian rhythm - plant & 33 & 60 & 0.000000 \\
\hline ko00944 & Flavone and flavonol biosynthesis & 15 & 21 & 0.000001 \\
\hline ko00360 & Phenylalanine metabolism & 26 & 57 & 0.000004 \\
\hline ko00350 & Tyrosine metabolism & 21 & 44 & 0.000019 \\
\hline ko00945 & Stilbenoid, diarylheptanoid and gingerol biosynthesis & 14 & 23 & 0.000027 \\
\hline ko00710 & Carbon fixation in photosynthetic organisms & 25 & 62 & 0.000062 \\
\hline ko01230 & Biosynthesis of amino acids & 58 & 206 & 0.000070 \\
\hline ko00010 & Glycolysis / Gluconeogenesis & 36 & 110 & 0.000124 \\
\hline ko01200 & Carbon metabolism & 63 & 238 & 0.000197 \\
\hline ko00250 & Alanine, aspartate and glutamate metabolism & 17 & 43 & 0.001956 \\
\hline ko00196 & Photosynthesis - antenna proteins & 10 & 19 & 0.002533 \\
\hline ko00260 & Glycine, serine and threonine metabolism & 22 & 66 & 0.003610 \\
\hline ko00750 & Vitamin B6 metabolism & 8 & 14 & 0.004533 \\
\hline ko00950 & Isoquinoline alkaloid biosynthesis & 11 & 24 & 0.004618 \\
\hline ko04626 & Plant-pathogen interaction & 44 & 171 & 0.004796 \\
\hline ko00052 & Galactose metabolism & 19 & 58 & 0.008664 \\
\hline ko00960 & Tropane, piperidine and pyridine alkaloid biosynthesis & 12 & 30 & 0.009569 \\
\hline
\end{tabular}


2

$\begin{array}{ll}\text { ko00941 } & \text { Flavonoid biosynthesis } \\ \text { ko04712 } & \text { Circadian rhythm - plant } \\ \text { ko00195 } & \text { Photosynthesis } \\ \text { ko00196 } & \text { Photosynthesis - antenna proteins } \\ \text { ko00945 } & \text { Stilbenoid, diarylheptanoid and gingerol biosynthesis } \\ \text { ko00940 } & \text { Phenylpropanoid biosynthesis } \\ \text { ko00360 } & \text { Phenylalanine metabolism } \\ \text { ko00592 } & \text { alpha-Linolenic acid metabolism }\end{array}$

\section{CK2 vs-B2}

ko00941 Flavonoid biosynthesis

ko04712 Circadian rhythm - plant

ko00360 Phenylalanine metabolism

ko00945 Stilbenoid, diarylheptanoid and gingerol biosynthesis

ko00940 Phenylpropanoid biosynthesis

ko04626 Plant-pathogen interaction

ko00130 Ubiquinone and other terpenoid-quinone biosynthesis

ko00592 alpha-Linolenic acid metabolism

CK2 vs-C2

ko00941 Flavonoid biosynthesis

ko04712 Circadian rhythm - plant

ko00945 Stilbenoid, diarylheptanoid and gingerol biosynthesis

ko00360 Phenylalanine metabolism

ko00940 Phenylpropanoid biosynthesis

ko04626 Plant-pathogen interaction

CK2-vs-D2

ko00941 Flavonoid biosynthesis

ko00360 Phenylalanine metabolism

ko00195 Photosynthesis

ko04712 Circadian rhythm - plant

ko00945 Stilbenoid, diarylheptanoid and gingerol biosynthesis

ko00940 Phenylpropanoid biosynthesis

ko00196 Photosynthesis - antenna proteins

ko00592 alpha-Linolenic acid metabolism

ko00400 Phenylalanine, tyrosine and tryptophan biosynthesis
0.007642

0.000000

0.000000

0.000003

0.000000

0.000000

0.000000

0.000000

0.000020

0.000659

0.003511

0.007769

56

0.000000

0.000000

0.000000

0.000001

0.000013

0.000194

0.000000 


\section{Figure 1}

Effects of different fruit bags on berry skin color. Bags with light transmittance of $50 \%$, $15 \%, 5 \%$ and 0 were performed at 45 days after flowering and designated as CK, A, B, $C$ and $D$, respectively. Unbagged berries as a control .

Effects of different fruit bags on berry skin color. B ags with light transmittance of $50 \%, 15 \%$ , $5 \%$ and 0 were performed at 45 days after flowering and designated as CK, A, B, C and D , respectively. Unbagged berries as a control . a Close-up views of wine grape cv. 'Marselan' berry fruit from different bagging treatments at S1, S2 and S3 . b Changes of a nthocyanin concentration at the three development stages. Error bars represent the \pm SE of three biological replicates, and the asterisks represent significant differences from the control, with $\mathrm{P}<0.05(*)$ or $\mathrm{P}<0.01(* *)$

a

S1
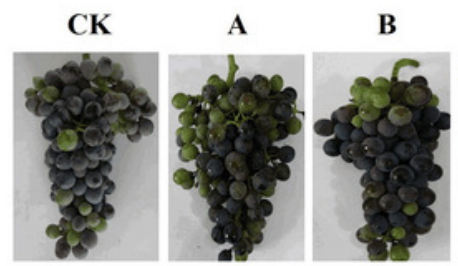

C

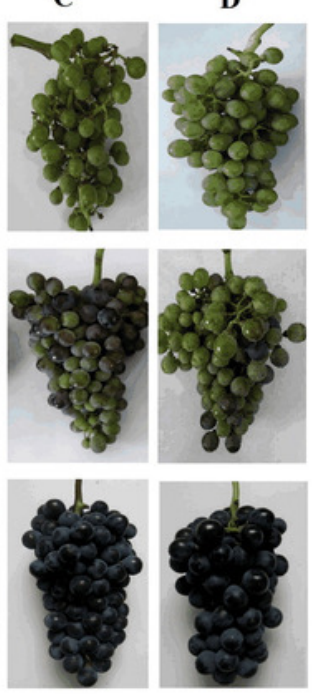

b

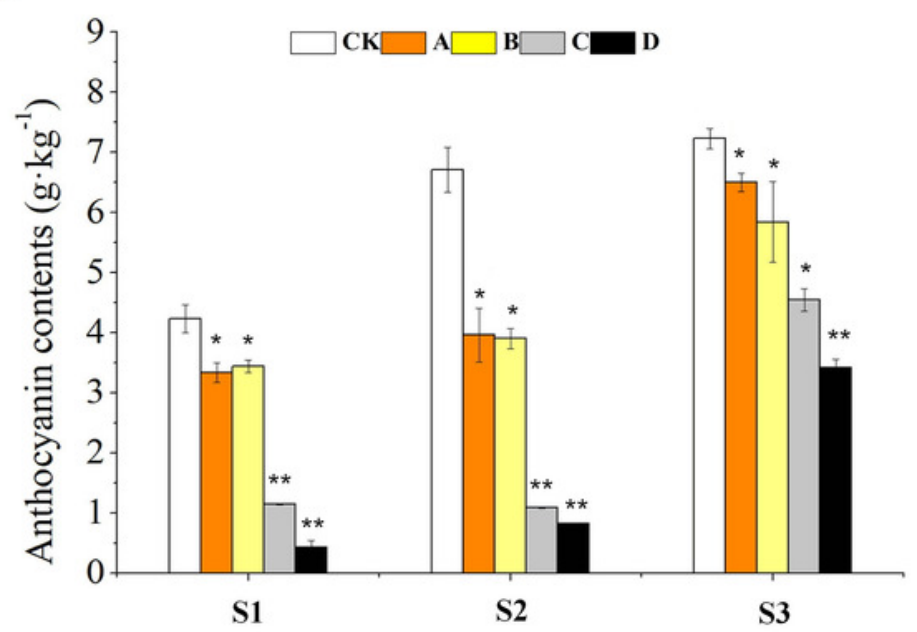




\section{Figure 2}

\section{Heatmap of the concentration of anthocyanins in different bagging treatments at $\mathrm{S} 1, \mathrm{~S} 2$} and S3 .
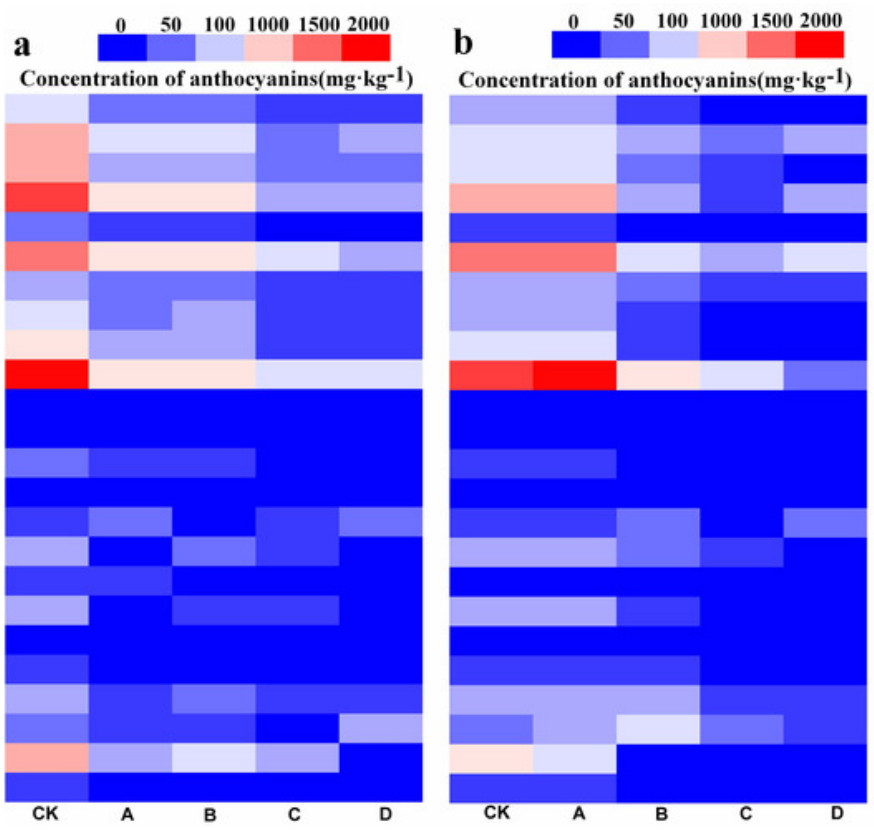

c

0 3001000200030004500

Concentration of anthocyanins $\left(\mathrm{mg} \cdot \mathrm{kg}^{-1}\right)$

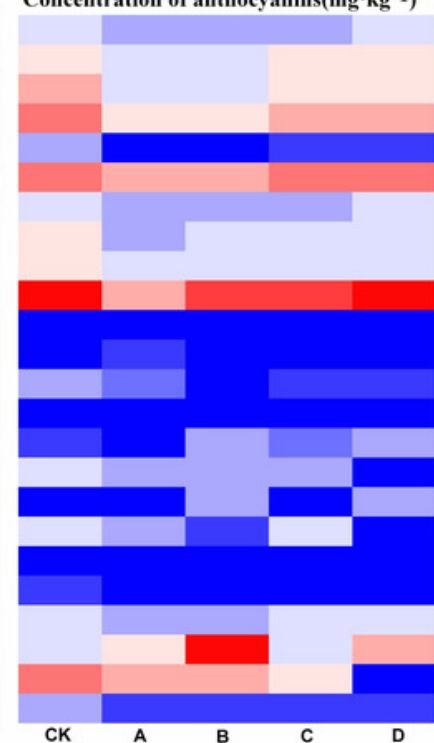

Cyanidin-3-0-glucoside

Peonidin -3-0-glucoside

Dephinidin -3-O-glucoside

Petunidin-3-0-glucoside

Cyanidin -3-0-acetylglucoside

Malvidin -3-O-glucoside

Peonidin -3-O-acetylglucoside

Dephinidin -3-0-acetylglucoside

Petunidin -3-O-acetylglucoside

Malvidin -3-O-acetylglucoside

Vinylformic acid adduct of malvidin-3-O-glucoside

Cyanidin -3-O-coumaroylglucoside (cis)

Cyanidin -3-O-coumaroylglucoside (trans)

Vinylformic acid adduct of malvidin-3-O-acetylglucoside

Peonidin -3-O-coumaroylglucoside (cis)

Peonidin -3-0-coumaroylglucoside (trans)

Dephinidin -3-0-coumaroylglucoside (cis)

Dephinidin -3-O-coumaroylglucoside (trans)

Peonidin -3-O-caffeoylglucoside

Petunidin -3-0-coumaroylglucoside (cis)

Petunidin -3-O-coumaroylglucoside (trans)

Malvidin -3-O-coumaroylglucoside (cis)

Malvidin-3-0-coumaroylglucoside (trans)

Malvidin -3-O-caffeoylglucoside 
Figure 3

The proportion of individual anthocyanins in total anthocyanin concentration from CK (panel a ) and D (panel b ) treatments at S3

a

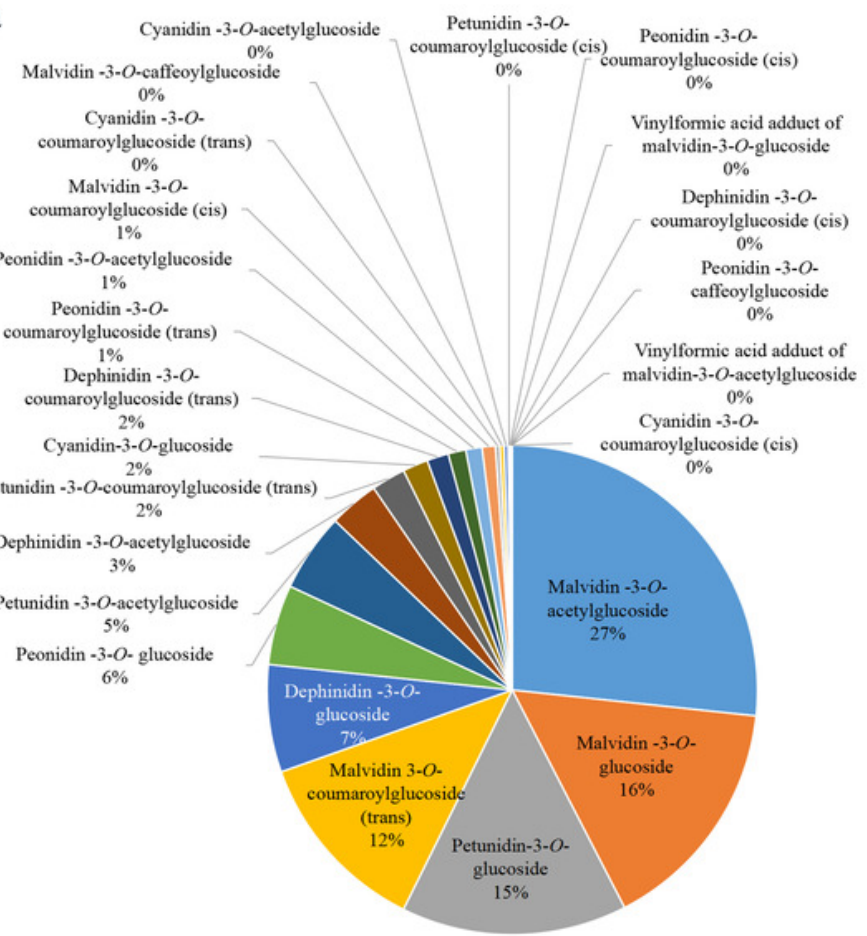

b

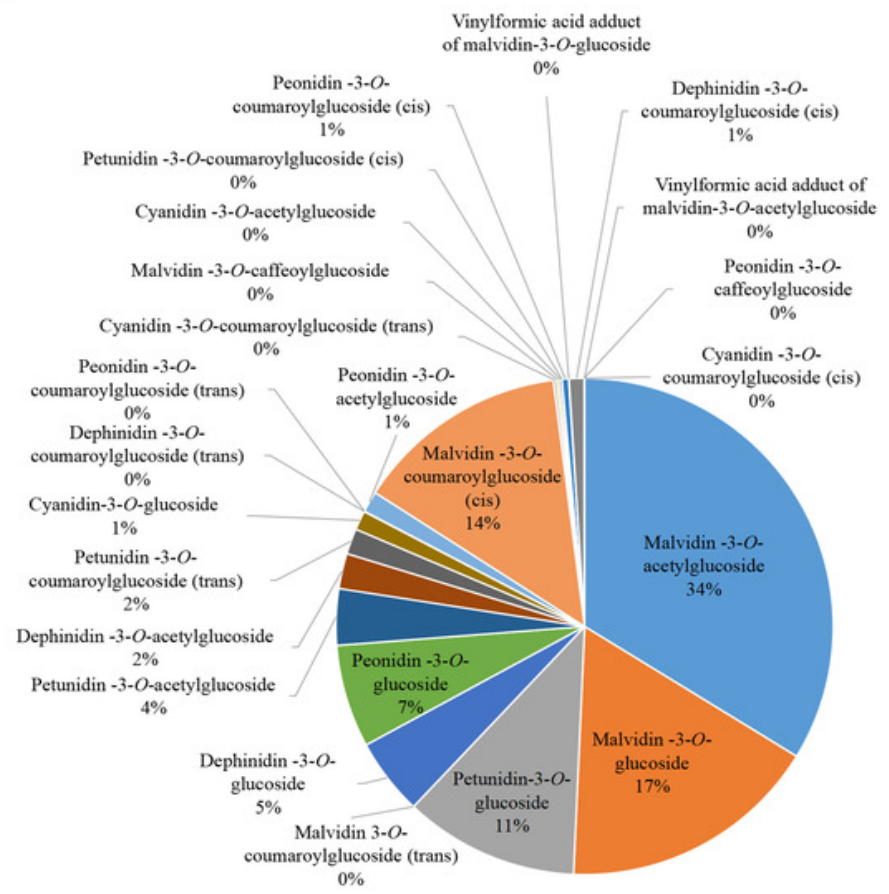




\section{Figure 4}

Heatmap of expressed genes assigned to anthocyanins synthesis in different bagging treatments.

Colors indicate expression values of the genes. Expression values of ten libraries are presented as FPKM normalized log2 transformed counts
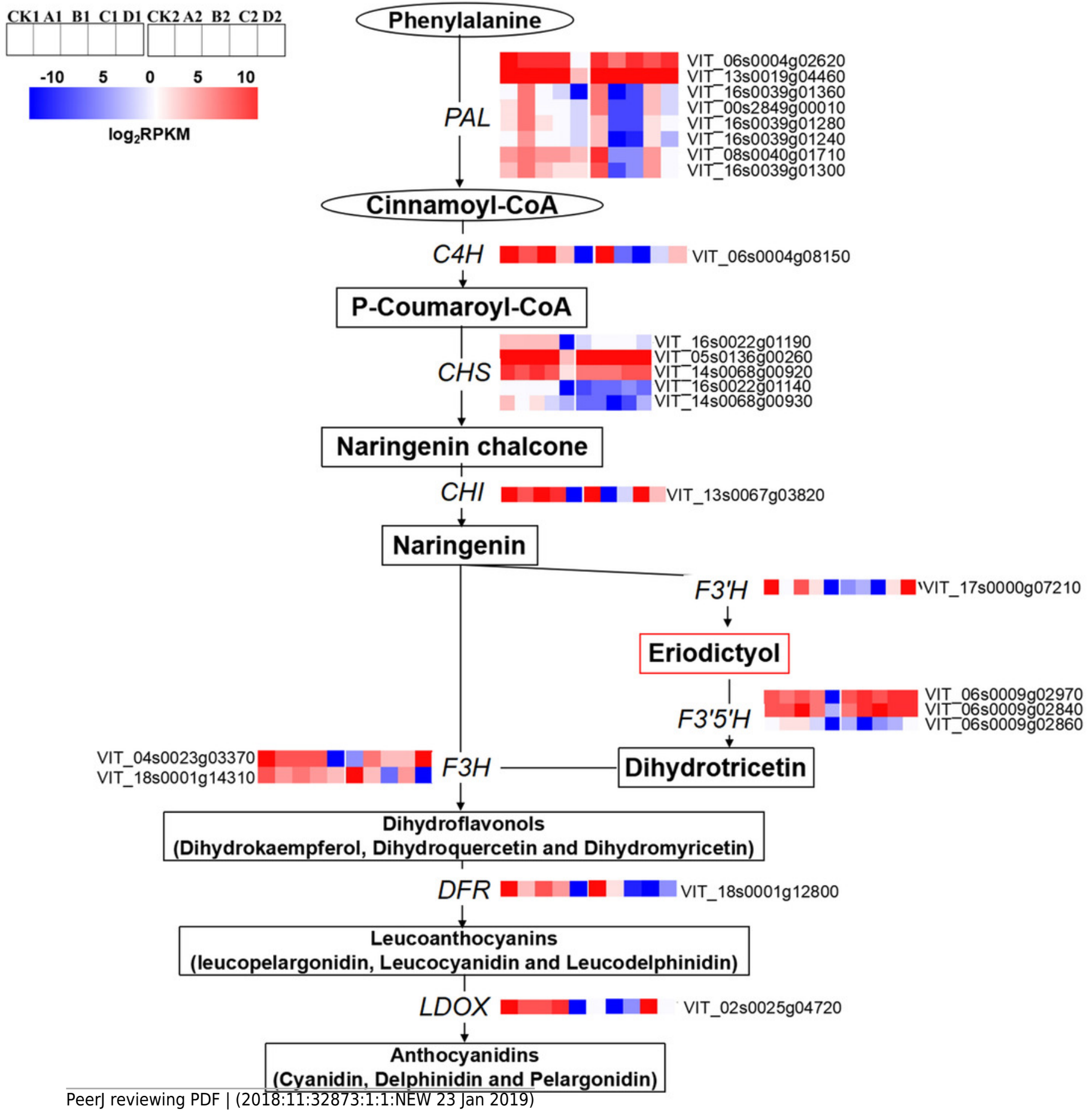


\section{Figure 5}

\section{Heatmap of MYB and basic helix-loop-helix (bHLH) transcription factors in different bagging treatments.}

Colors indicate expression values of the genes. Expression values of ten libraries are presented as FPKM normalized log2 transformed counts

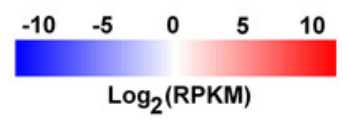

a

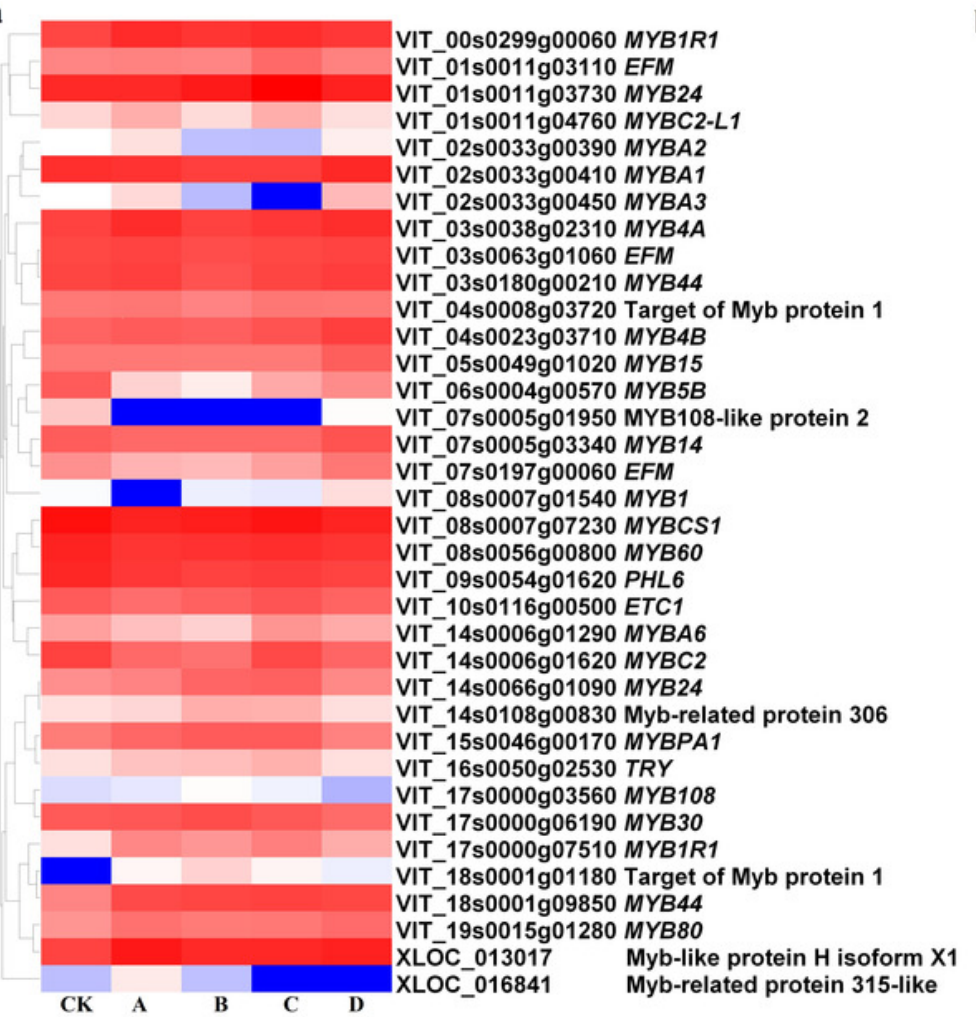

b

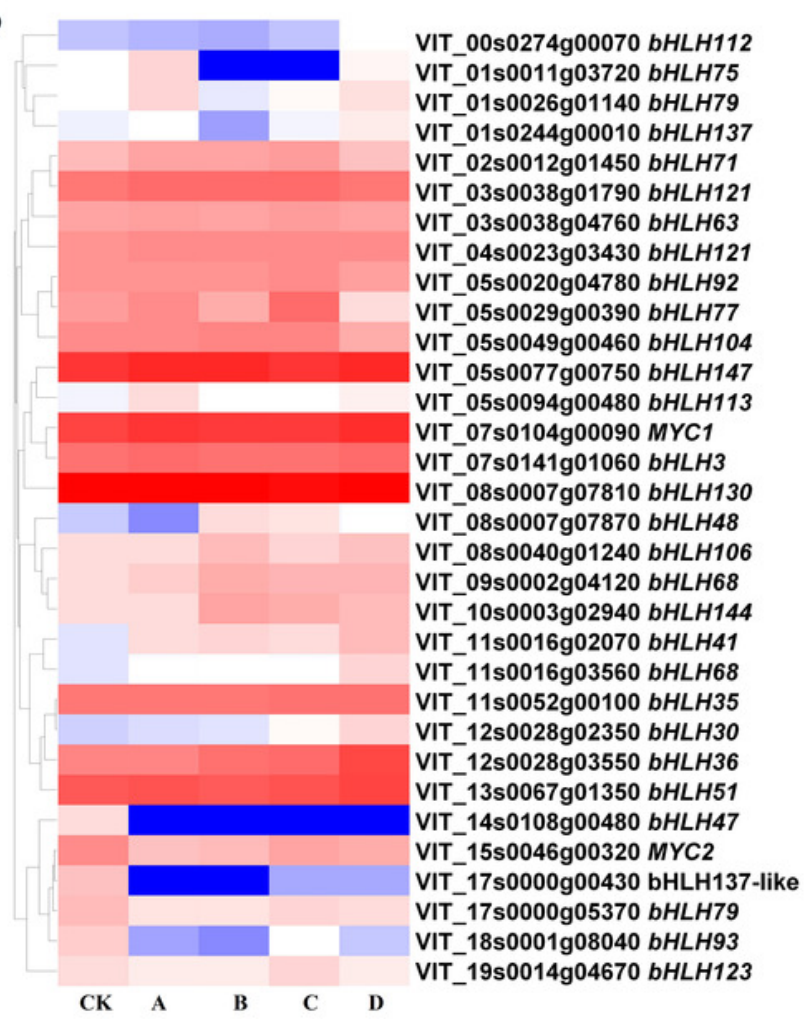




\section{Figure 6}

qRT-PCR validation of 4 candidate genes related to anthocyanins synthesis from the different bagging treatments.

qRT-PCR validation of 4 candidate genes related to anthocyanins synthesis from the different bagging treatments. The left $y$-axis indicates relative gene expression levels were determined by qRT-PCR and analyzed using $2^{-\triangle \Delta C T}$ Method. The $x$-axis indicates different treatments. All qRT-PCR for each gene used three biological replicates, with three technical replicates per experiments. Error bars indicate $\pm \mathrm{SE}$, and the asterisks represent significant differences from the control, with $\mathrm{P}<0.05(*)$ or $\mathrm{P}<0.01(* *)$ 


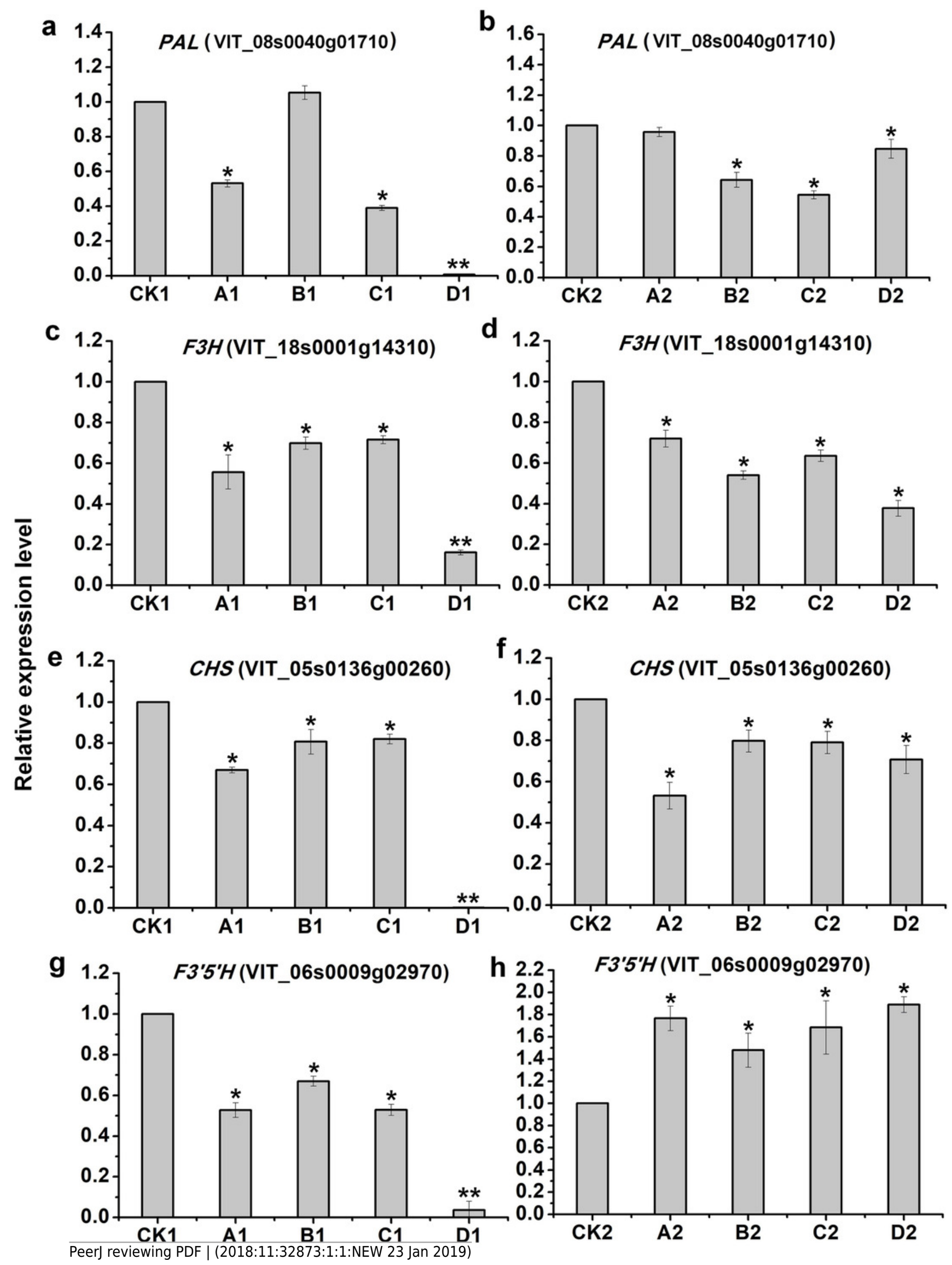

